PNNL-13321

\title{
Evaluation of SAFT/T-SAFT Technology for the Inspection of Hanford's Double Shell Waste Tank Knuckle Regions
}

\author{
A. F. Pardini \\ A. A. Diaz
}

September 2000

Prepared for the U.S. Department of Energy under Contract DE-AC06-76RLO 1930

This work is funded by the Office of Science and Technology within the Energy's Office of Environmental Management under the Tanks Focus Area Program 


\title{
DISCLAIMER
}

This report was prepared as an account of work sponsored by an agency of the United States Government. Neither the United States Government nor any agency thereof, nor Battelle Memorial Institute, nor any of their employees, makes any warranty, express or implied, or assumes any legal liability or responsibility for the accuracy, completeness, or usefulness of any information, apparatus, product, or process disclosed, or represents that its use would not infringe privately owned rights. Reference herein to any specific commercial product, process, or service by trade name, trademark, manufacturer, or otherwise does not necessarily constitute or imply its endorsement, recommendation, or favoring by the United States Government or any agency thereof, or Battelle Memorial Institute. The views and opinions of authors expressed herein do not necessarily state or reflect those of the United States Government or any agency thereof.

\author{
PACIFIC NORTHWEST NATIONAL LABORATORY \\ operated by \\ BATTELLE \\ for the \\ UNITED STATES DEPARTMENT OF ENERGY \\ under Contract DE-AC06-76RL01830
}

Printed in the United States of America

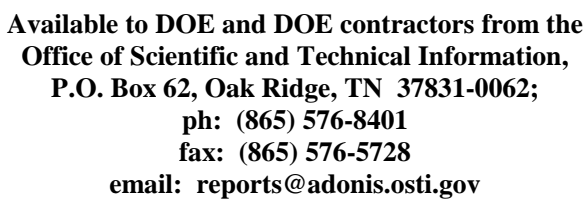

Available to the public from the National Technical Information Service, U.S. Department of Commerce, 5285 Port Royal Rd., Springfield, VA 22161 ph: (800) 553-6847 fax: $(703) 605-6900$

email: orders@ntis.fedworld.gov

online ordering: http://www.ntis.gov/ordering.htm 


\title{
FINAL REPORT
}

\section{Evaluation of SAFT/T-SAFT Technology for the Inspection of Hanford's Double Shell Waste Tank Knuckle Regions}

\author{
A. F. Pardini \\ A. A. Diaz
}

September 2000

Prepared for the U.S. Department of Energy

under Contract DE-AC06-76RLO 1930

This work is funded by the Office of Science and Technology within the Energy's Office of Environmental Management under the Tanks Focus Area Program, Technical Task Plan RL30WT21 
PNNL-13321 
PNNL-13321

\section{Summary}

The need to examine the knuckle region of Hanford's double shell tanks has received considerable attention over the last few months. Commercial, off-the-shelf systems to examine the knuckle are not available. Preliminary tests at the Pacific Northwest National Laboratory (PNNL) in FY 1999 indicated that a technology utilizing ultrasonics could provide a solution to the knuckle examination problem. In FY 2000 PNNL embarked on a study to provide evidence that the ultrasonic technology had the capability to detect and size stress corrosion cracks in the knuckle region of the double shell tanks.

Analysis of the examination results conducted at PNNL provide engineering data strongly supporting a proof-of-principle concept for utilizing a combination of pulse-echo Synthetic Aperture Focusing Technique (SAFT) and tandem-SAFT (T-SAFT) inspection methodologies. These methods can be applied to the problem of flaw detection, localization, and sizing in Hanford's double shell waste tank knuckle region and beyond.

The work described in this report includes a successful demonstration of the $70^{\circ}$ shear wave inspection technique for detection and localization of reflectors in two different geometrical cases. In the first case, that of a flat plate, results showed that the inspection protocol on a 25.4-mm thick carbon steel plate over distances ranging from approximately $889 \mathrm{~mm}$ to $1067 \mathrm{~mm}$ provided an effective method for examination. In the second case, that of a mockup of the double shell tank knuckle region, results clearly indicate that the inspection protocol was capable of detecting, localizing and sizing of machined reflectors under more realistic conditions.

In the case of the flat plate, signal response amplitudes varied with the addition of weldment geometry between the transmitting transducer and machined reflectors. Enhancements provided by SAFT processing appear to provide an effective means for analyzing those signal response amplitudes and developing an optimized inspection protocol for the knuckle region and beyond. Data acquired from examinations in the laboratory show that the inspection technique is capable of detecting and localizing machined reflectors of the order of $5.1 \mathrm{~mm}$ deep, from long path lengths and through a weld.

In the case of the mock-up examinations, data clearly indicated the successful demonstration of the $70^{\circ}$ shear wave inspection technique for detection and localization of reflectors in a more realistic configuration. Once again, signal response amplitudes varied with the addition of the knuckle geometry curvature between the transmitting transducer and machined reflectors; however, enhancements provided by SAFT processing provided the means to compensate for these adverse conditions. The additional capability of utilizing T-SAFT for improved depth sizing of the machined reflectors was shown in this work as well. 
The examination frequency of 3.5 megahertz $(\mathrm{MHz}$ ) provided good resolution and suitable penetration over the long path lengths. Pulse-echo data acquired from examinations on the mock-up showed that the inspection technique is capable of detecting and localizing machined reflectors, over long path lengths and around the knuckle region curvature. Sizing of the reflector depth was not examined in great detail; however, the T-SAFT data indicated the ability to size shallow machined reflectors (with depths greater than $4.6 \mathrm{~mm}$ ) within the established tolerance of $\pm 1.27 \mathrm{~mm}$ presently in effect.

The inspection system used to acquire data in this study was not optimal for ultrasonically long-range, attenuative inspection environments. With off-the-shelf electronics and transducers, the inspection techniques evaluated in this study were successfully employed and demonstrated even under such adverse conditions as propagating the sound field through a weldment and around the curved knuckle region. As laboratory examination techniques, the pulse-echo and tandem-SAFT inspection methodologies implemented here are sufficient for the purposes of demonstrating the feasibility for developing a prototype system based on these technologies. However, the entire inspection protocol must be improved in order to efficiently utilize these techniques in combination with a field-ready inspection system. In order to take the proposed inspection protocol to the next level, a number of key elements in the system will require optimization and further refinement.

The work described in this report was funded and supported by the U.S. Department of Energy's (DOE) Tanks Focus Area (TFA) Safety program under the coordination of Mike Terry. The TFA provides science and technology solutions to safely and efficiently remediate waste stored in underground storage tanks at five DOE sites. 
PNNL-13321

\section{Acknowledgments}

The authors acknowledge the contributions of the following PNNL staff members: Mr. George Schuster for his assistance in software configuration and T-SAFT modifications, Dr. Steven Doctor for his guidance and direction in path forward planning, Mr. James Alzheimer for modifications to the double shell tank mockup, and Mrs. Twilla Knowles and Mr. Todd Samuel for administrative assistance.

The authors would also like to acknowledge the contributions of Mr. Mike Terry, Tanks Focus Area Safety Technical Integration Manager, and Mr. Chris Jensen from $\mathrm{CH}^{2} \mathrm{M}$ Hill Hanford Group for problem definition and reviews. 
PNNL-13321 


\section{Table of Contents}

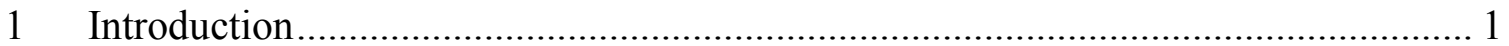

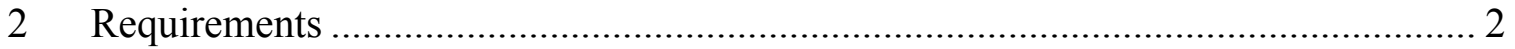

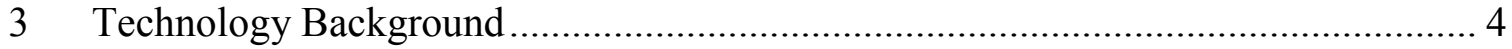

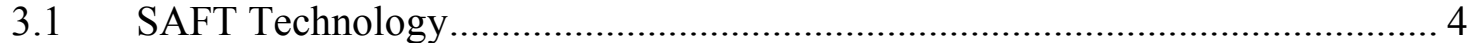

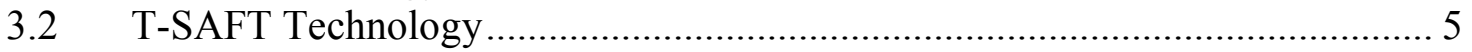

3.3 Signal Processing Parameters ………………................................................ 7

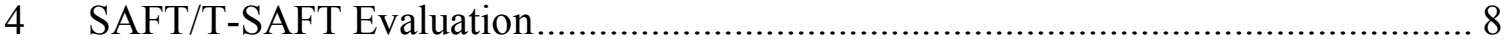

$4.1 \quad$ Flat Plate Analysis .................................................................................... 8

4.1.1 Flat Plate Simulation Using IMAGINE3D Software................................ 8

4.1.2 Flat Plate Geometry and Reflectors ................................................... 9

4.1.3 Data Acquisition Configuration ......................................................... 9

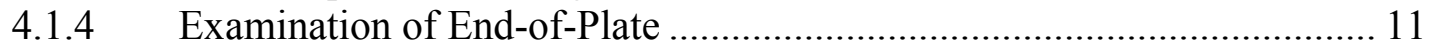

4.1.5 Examination of Sawcuts .................................................................. 13

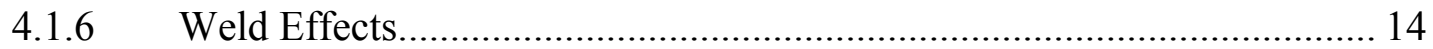

4.1.7 Summary of Flat Plate Examinations ………….................................... 15

4.2 Mock-up Analysis ........................................................................................ 15

4.2.1 Mock-up Simulation Using IMAGINE3D Software …………………..... 15

4.2.2 Mock-up Geometry and Reflectors..................................................... 16

4.2.3 Data Acquisition Configuration ............................................................... 17

4.2.4 Examination of End-of-Knuckle on Mock-up .......................................... 19

4.2.5 Examination of Sawcuts ................................................................. 20

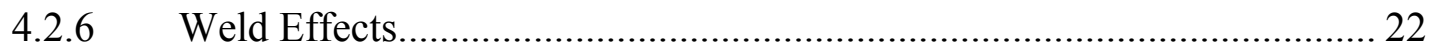

4.2.7 Summary of Mock-up Examinations ....................................................... 22

5 Deployment Methods for SAFT/T-SAFT Technology......................................... 22

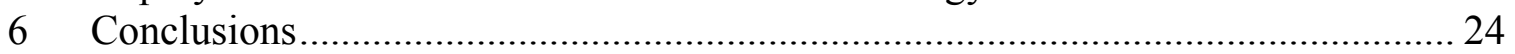

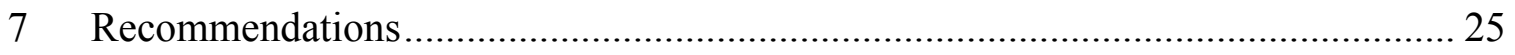

7.1 Recommendations for Enhancing SAFT Processing Utility ............................ 25

7.2 Recommendations for Enhancing Data Acquisition System Utility................. 25

7.3 Recommendations for Enhancing Data Analysis and Visualization ................ 26

7.4 Recommendations for Prototype Examination System Development, Field

Testing and Performance Demonstration.............................................................. 26

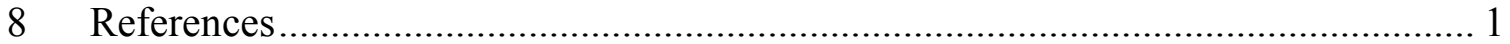




\section{List of Figures}

Figure 2.1 Planar Crack on Primary Tank Inside Diameter ......................................2

Figure 2.2 Examination Parameters in Knuckle Region .............................................

Figure 3.1 T-Shaft Scanning Transducer Configuration .........................................6

Figure 3.2 Side View Showing V Paths .............................................................6

Figure 4.1 IMAGINE3D Simulation of Flat Plate Inspection Using $70^{\circ}$ Shear Waves......................................................................8

Figure 4.2 Sawcut-12.7-mm Deep by 71.1-mm Long ............................................

Figure $4.3 \quad$ Flat Plate Configuration ...................................................................

Figure 4.4 Flat Plate Test Configuration ............................................................... 10

Figure 4.5 Evolution of Wave Packet as a Function of Path Length from End of Plate ..........................................................11

Figure 4.6 SAFT-Processed B-Scan Side View and C-Scan Top View of End Plate .......................................................................12

Figure 4.7 A-Scans of Small and Large Sawcuts ..................................................13

Figure 4.8 SAFT-Processed B-Scan Side View and C-Scan Top View of End Plate ............................................................................14

Figure 4.9 Scanning of Flat Plate with Weld ........................................................14

Figure 4.10 SAFT-Processed C-Scan Comparison of 5.1-mm Sawcut Through Weld .................................................................................15

Figure 4.11 IMAGINE3D Simulation of Knuckle Region .......................................16

Figure 4.12 Mockup Knuckle Showing Sawcuts ....................................................16

Figure 4.13 Scanning System on Mockup.............................................................17

Figure 4.14 T-SAFT Scanning Configuration on Mockup ..........................................18

Figure 4.15 Comparison of A-Scan Wave Packets Between the Flat Plate and the Mockup ..............................................................19

Figure 4.16 SAFT-Processed B-Scan and C-Scan Images of the End of the Knuckle Plate Mockup .........................................................20

Figure 4.17 SAFT-Processed B-Scan and C-Scan Images of 4.6-mm Sawcut on Mockup ...................................................................................20

Figure 4.18 SAFT-Processed B-Scan and C-Scan Images of 2-mm Sawcut on Mockup ............................................................................21

Figure 4.19 SAFT-Processed B-Scan and C-Scan Images of 4.6-mm Sawcut on Mockup

Figure 5.1 SAFT/T-SAFT Deployment Method .23

\section{Tables}

Table 2.1 Sizing Requirements .3 
PNNL-13321

\section{Introduction}

One of the key elements in ensuring the integrity of the Hanford's Double-Shell Tanks (DSTs) is the examination of the knuckle region of the primary tank. This examination poses a significant technical challenge because the area that requires examination is not accessible using conventional measurement techniques. The Pacific Northwest National Laboratory (PNNL) has offered a possible solution to this problem. Utilizing an ultrasonic technique known as Synthetic Aperture Focusing Technique (SAFT), PNNL proposes to introduce sound waves from above the knuckle region where access is readily achieved. The sound is divergent in nature and propagates around the knuckle and along the bottom of the waste tank. The SAFT technique should provide a detection and location method for cracks in the knuckle region. An advanced nondestructive evaluation (NDE) method known as Tandem-SAFT or T-SAFT utilizes two transducers in a pitchcatch mode to characterize the detected crack. T-SAFT has the ability to accurately size the crack both in length and depth.

This report documents work performed at the PNNL for evaluating the effectiveness of advanced ultrasonic examination techniques coupled with SAFT signal processing methodologies used for the detection and characterization of indications in the knuckle region and beyond. Two sample specimens were used in this study, a flat plate and a larger-scale sectioned mock-up of the region of interest in the double wall tank configuration. These samples were fabricated to simulate realistic conditions and provide various reflectors as a function of position and dimensions in and about the area of interest. The primary focus of this work is to provide information to Tank Focus Area (TFA) staff regarding the utility, effectiveness and performance of these ultrasonic techniques as related to the detection and sizing of indications in the tank wall along and beyond the knuckle region.

This document has been divided into six major sections. Section 2 describes the requirements that must be met for the successful examination of the knuckle region of the double shell waste tanks. Section 3 provides background information on SAFT and T-SAFT technology. Section 4 provides the evaluation of SAFT and T-SAFT as promising technology for the examination of the double shell waste tank knuckles. Section 5 provides deployment methods that were evaluated and the preferred deployment method for the SAFT/T-SAFT technology. Section 6 provides conclusions based on this study and Section 7 provides recommendations for future work. 


\section{Requirements}

To assure that the DSTs at Hanford maintain their structural integrity, an inspection plan was developed and implemented (Pfluger 1994). This inspection plan describes the ultrasonic testing (UT) system, the qualification of the equipment and procedures, field inspection readiness, DST inspections, and post-inspection activities. The plan also provides the basis for the flaw characterization requirements. Utilizing this information, a Functions and Requirements (F\&R) document is being developed by the PNNL to define a system capable of reliably examining the knuckle region of the primary waste tank. Specifically, PNNL is chartered with developing a Nondestructive Examination (NDE) system to examine the knuckle region. PNNL examined this inspection challenge and determined that the best approach would be based on using SAFT and an advanced NDE sizing technique utilizing tandem transducers known as T-SAFT.

The flaw characteristics of interest are planar flaws located in the knuckle region emanating from the inside surface of the tank. This region contains the highest stress point of the entire primary steel tank (Shurrab et al., 1991). Examinations shall concentrate on cracks that are caused by stress corrosion and are circumferentially oriented. Figure 2.1 provides a graphical example of a planar-type stress corrosion crack that is of interest.

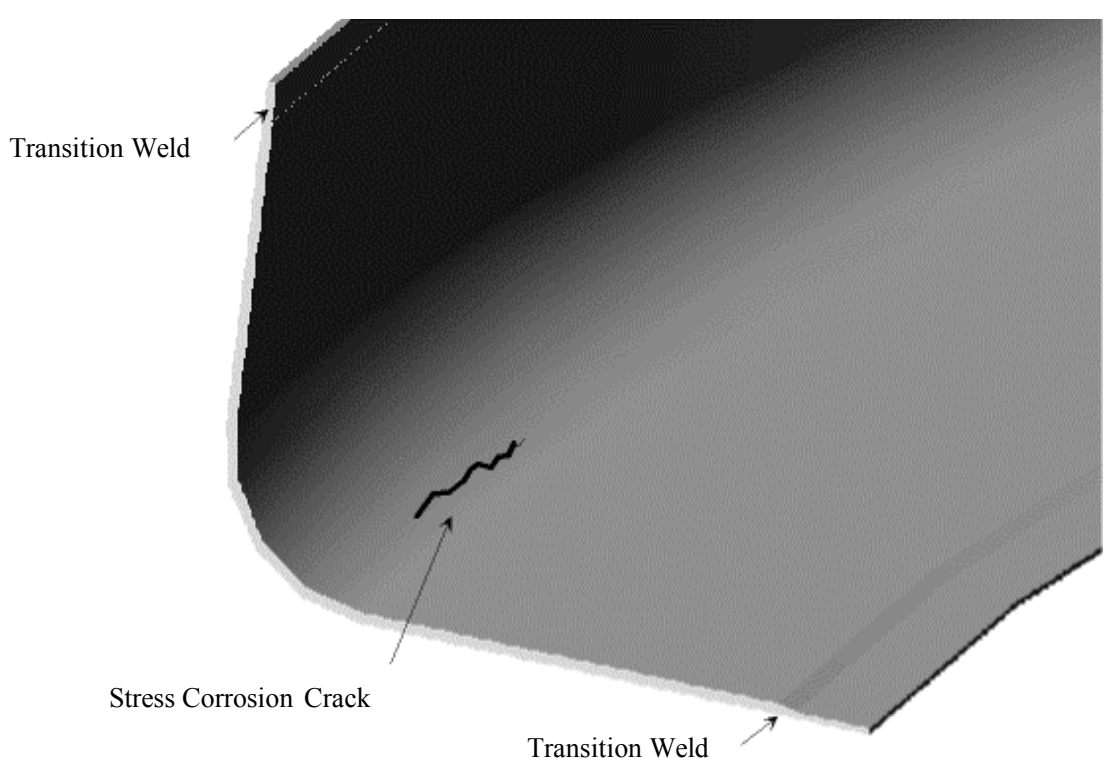

Figure 2.1 Planar Crack on Primary Tank Inside Diameter

The flaw characterization requirements (Pfluger 1995) stipulate the minimum dimension that is to be characterized and the accuracy requirements. Circumferential cracks emanating from the inside surface of the primary tank shall be detected when the crack depth is greater than $0.2 \mathrm{t}$ where $\mathrm{t}$ is the thickness of the knuckle region. Characterization of the crack shall be in accordance with Table 2.1 . 
PNNL-13321

Table 2.1 Sizing Requirements

\begin{tabular}{|c|c|c|}
\hline Condition & $\begin{array}{c}\text { Minimum Dimension } \\
\text { To be Characterized }^{(\mathbf{1})}\end{array}$ & Accuracy \\
\hline Cracks (circumferential) & $305-\mathrm{mm}$ long $\times 0.2 \mathrm{t}$ deep & $\pm 1.27 \mathrm{~mm}$ (depth) \\
& & $\pm 12.7 \mathrm{~mm}$ (length) \\
\hline
\end{tabular}

${ }^{1}$ Nominal tank wall thickness is t.

The SAFT/T-SAFT system shall be capable of detecting planar flaws located in the knuckle region of the primary tank. The knuckle region as shown in Figure 2.2 describes the inspection areas. The inspection area begins just above the construction weld on the vertical portion of the tank and extends to just past the transition weld located on the tank bottom. Locations of construction welds vary depending on which tank farm is being inspected.

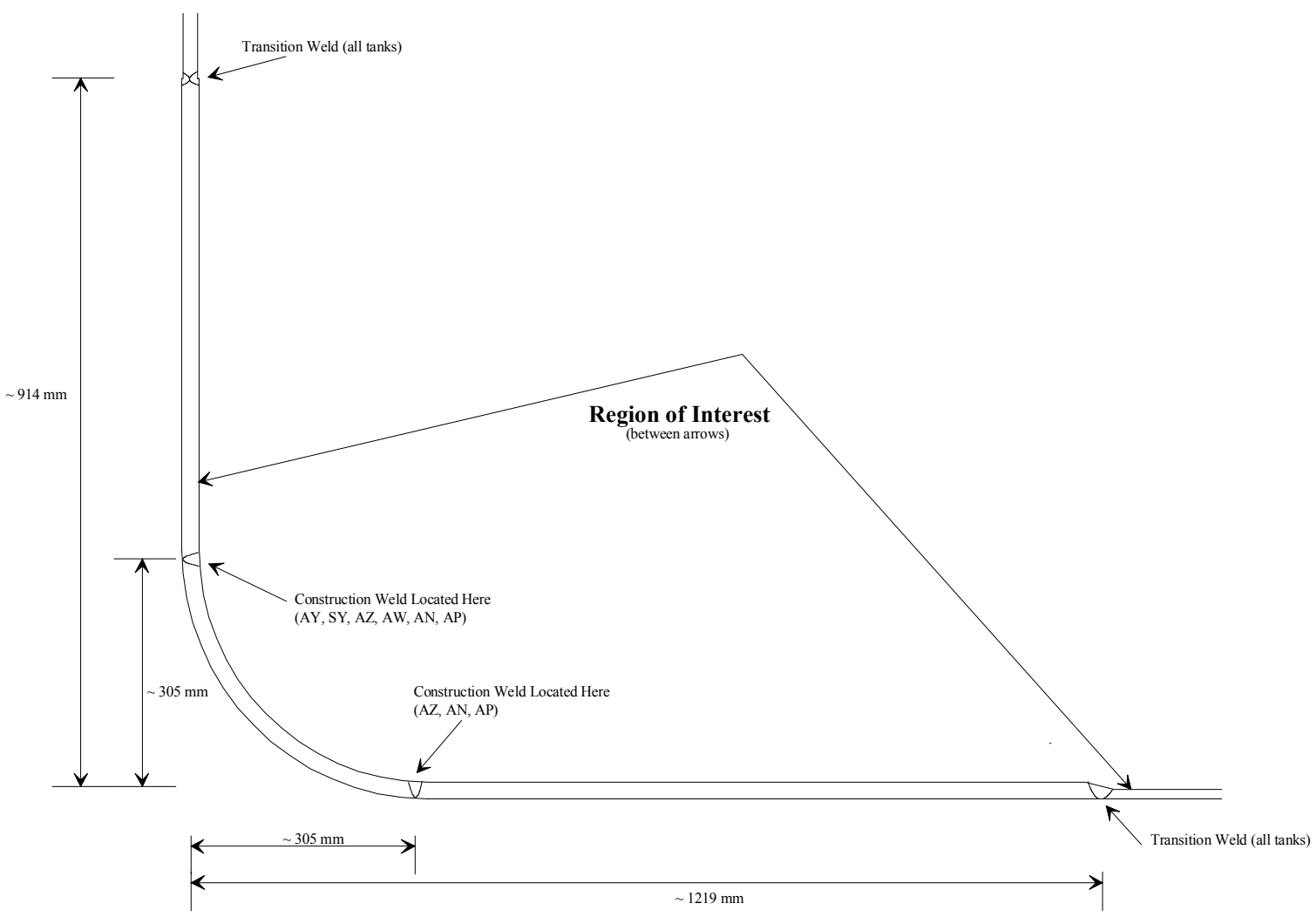

Figure 2.2 Examination Parameters in Knuckle Region 
PNNL-13321

\section{Technology Background}

\subsection{SAFT Technology}

SAFT technology is able to provide significant enhancements to the inspection of materials when using unfocused ultrasonic transducers where the attenuative effects of path length, material noise, and sound beam divergence are evident. The resolution of all imaging systems is limited by the effective aperture area, that is, the area over which data can be detected, collected, and processed. SAFT is an imaging method, which was developed to overcome some of the limitations imposed by large physical transducer apertures, and has been successfully applied in the field of ultrasonic testing. Relying on the physics of ultrasonic wave propagation, SAFT is a very robust technique.

"Synthetic aperture focusing" refers to a process in which the focal properties of a largeaperture focused transducer are synthetically generated from data collected over a large area using a small transducer with a divergent sound field (Hall et al., 1988). The processing required to focus this collection of data has been called beam-forming, coherent summation, or synthetic aperture processing. The resultant image is a full-volume, high resolution, and high signal to noise ratio (SNR), focused characterization of the inspected area.

Utilizing the pulse-echo configuration for typical data collection, the transducer was positioned on the surface of the specimen, and radio frequency (rf) ultrasonic data were collected. As the transducer was scanned over the surface of the specimen, the A-scan records (rf waveform) were amplified, filtered, and digitized for each position of the transducer. Each reflector produced a collection of echoes in the A-scan records. The unprocessed rf data sets were then post-processed using the SAFT algorithm, and invoking a variety of full beam angle values (between $1^{\circ}$ and $6^{\circ}$ ) in an attempt to optimize the spatial averaging enhancement.

If the reflector is an elementary single point reflector, then the collection of echoes will form a hyperbolic surface within the data-set volume. The shape of the hyperboloid is determined by the depth of the reflector in the specimen and the velocity of sound in the specimen. This relationship between echo location in the series of A-scans and the actual location of the reflectors within the specimen makes it possible to reconstruct a high-resolution, high SNR focused image from the acquired raw data.

If the scanning and surface geometries are well known, it is possible to accurately predict the shape of the locus of echoes for each point within the test object. The process of coherent summation for each image point involves shifting a locus of A-scans, within a regional aperture, by predicted time delays and summing the shifted A-scans. This process may also be viewed as performing a spatial matched filter operation for each point within the volume to be imaged. Each element is then averaged by the number of points that were summed to produce the final processed value. If the particular location correlates with the elementary point response hyperboloid, then the values summed will 
be in phase and produce a high-amplitude result. If the location does not correlate with the predicted response, then destructive interference will take place and the spatial average will result in a low amplitude value, thus reducing the noise level to a very small value.

All SAFT processing software was contained in and invoked on a personal computer (PC) workstation. Images presented here for analysis were generated after post processing the rf data and projecting the images in a two-dimensional planar view. The SAFT imaging software provides the user the capability to view the entire ultrasonic data set (three-dimensional array of points) in two-dimensional slices. The software provides a platform for viewing color-enhanced composite images that depict slices of the threedimensional array in the $\mathrm{X}-\mathrm{Y}$ plane (C-scan view), the $\mathrm{Y}-\mathrm{Z}$ plane (B-Scan end-view) and $\mathrm{X}-\mathrm{Z}$ plane (B-scan side view).

\subsection{T-SAFT Technology}

A single transducer in pulse-echo configuration works well for location and detection of a defect but may provide ambiguous results for sizing of the defect in the knuckle region and beyond due to the lack of a tip-diffracted signal. The tandem configuration reduces the ambiguities and improves sizing of vertical defects. In this study, the pulse-echo configuration coupled with SAFT processing will used for detection and localization of a defect, and this information will be used to optimize the spatial positioning for a tandem configuration to be implemented for sizing of the defect.

Fundamentally, the tandem SAFT (T-SAFT) configuration used in this study provides a uniform illumination of the vertical object plane. The central ray of the transmitter's divergent beam is always centered on the receiving transducer by scanning the transmitter synchronous but in the opposite direction of the receiver. At the completion of each pass of transmitter and receiver, the two-transducer configuration is incremented so that a rectilinear pattern is obtained. Since a more uniform illumination of the vertical object plane is possible, the vertical extent of a defect can be accurately measured. When both paths are collected and processing occurs beyond the far surface, the result is a real and conjugate image. Sizing is accomplished by measuring the extent of the real and conjugate images and dividing the resultant value by two. In the T-SAFT mode, the transmitter initially starts in front of the receive transducer. Both transducers are scanned equal distances but in opposite directions as shown in Figure 3.1 (top view) and Figure 3.2 (side-view). 


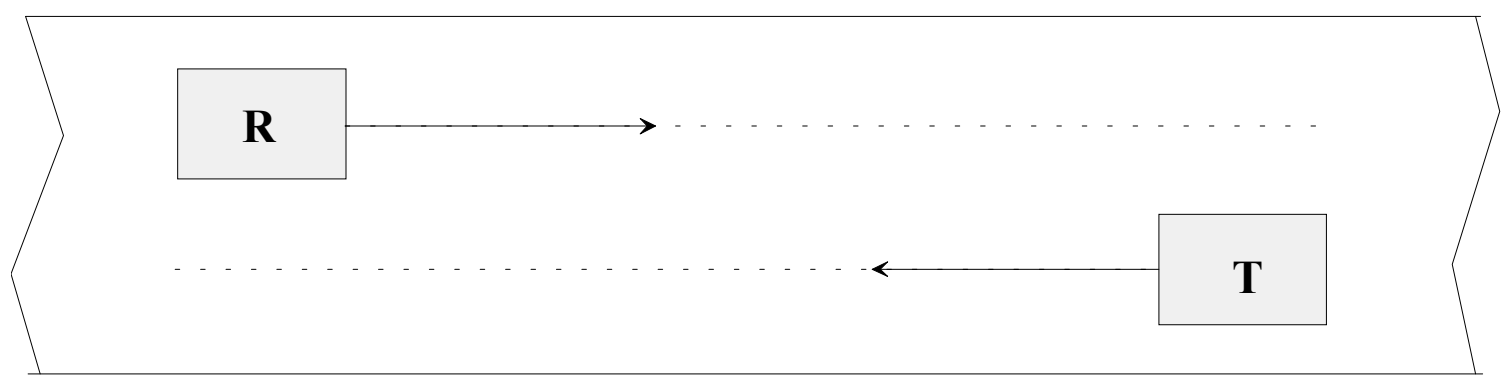

a) Initial Position of Transducers for T-SAFT Scanning (Top View)

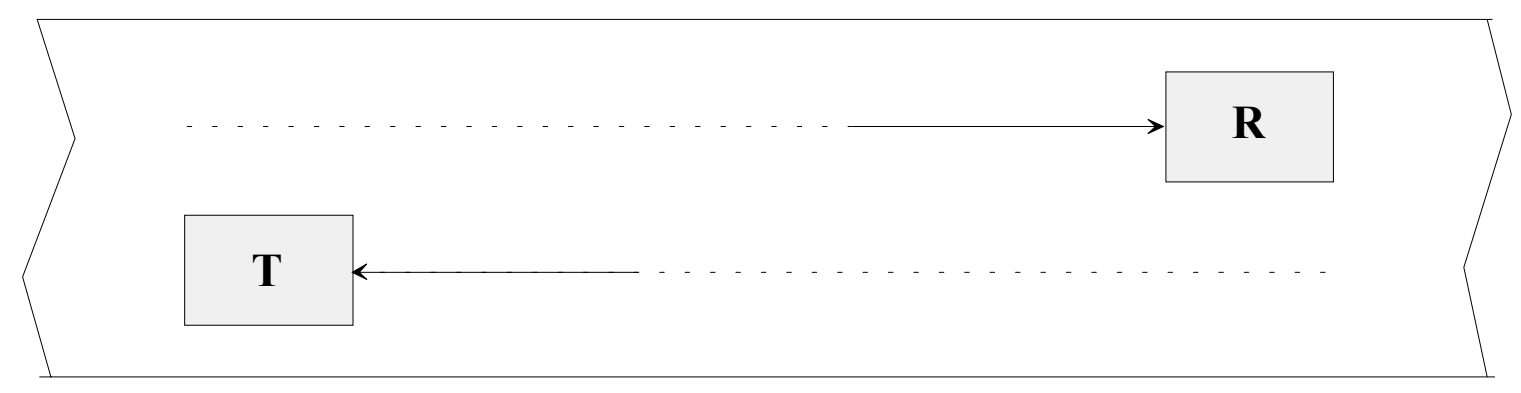

b) Final Position of Transducers for a Single Pass in T-SAFT Scanning Mode (Top View)

Figure 3.1 T-SAFT Scanning Transducer Configuration

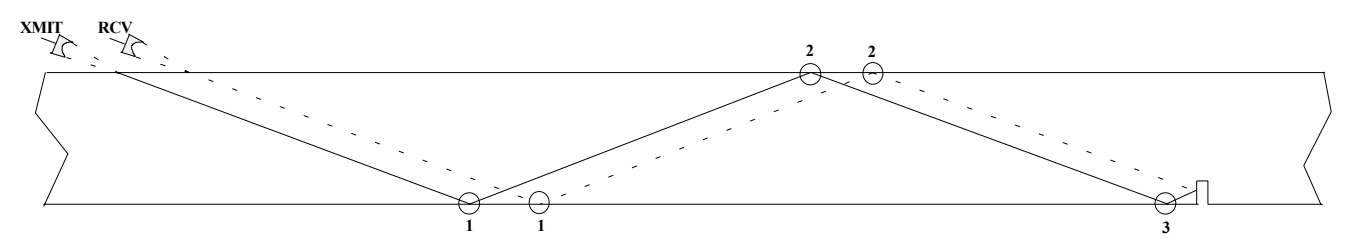

Figure 3.2 Side View Showing V Paths

Tandem image analysis uses techniques similar to those of pulse-echo analysis. Defects may be categorized as volumetric, planar, or crack. The primary difference between the tandem and pulse-echo image is that the tandem image of a crack presents the entire cross section of the crack and not just the corner-trap and tip-diffracted echoes. Often, the tipdiffracted echo is very illusive because of the weak nature of the tip-diffracted echo compared to the very strong corner-trap echo; and without a tip-diffracted echo, the vertical extent of a crack is difficult to estimate. 
The signal-to-noise ratio of a tandem image is often much superior to that of a pulse-echo image, because a separate receiver eliminates noise caused by the initial pulse, the near surface interface, and the specular backscatter from the material structure under examination. Tandem image indications are vertical in appearance, as opposed to the slanted (projected) appearance of a pulse-echo image. The location of the indication within the image space is influenced by the material thickness, velocity, and refracted angle. In this study, the wall thickness in the area of the knuckle region is assumed to be accurately known. The SAFT/T-SAFT algorithm implemented at PNNL assumes isotropic and homogeneous material with respect to acoustic velocity; that is, the calculations performed by the SAFT processing make the approximation that the velocity is constant throughout the material. Also, the algorithm requires that the acoustic velocity of the material under test be known to some degree of accuracy. This is the case with the carbon steel specimens used in this study.

\subsection{Signal Processing Parameters}

The SAFT signal processing algorithm requires the entry of multiple parameters that affect the resultant processed output. This section describes the basis for using the various signal processing parameters employed on the raw data. The SAFT processing software requires the operator to enter pertinent information associated with the transducer(s) used, the acoustic modality, refracted angle, frequency, geometric information, material property information, sampling information and so forth. These parameters vary in their significance with regard to their affect upon the resultant processed output.

In this study, $70^{\circ}$ shear waves were implemented as the primary inspection modality. The transducers used were circular-contact, flat piston radiators, $12.7 \mathrm{~mm}$ in diameter with nominal center frequencies of 3.5 megahertz $(\mathrm{MHz})$. The transducers were affixed to wedges that provided the correct incident angle for propagation of $70^{\circ}$ shear waves in the material under test. The full beam angle (entered in degrees) affects the processed image and determines the size of the synthetic aperture to be used when SAFT processing is performed. Typically, small beam angles are used initially to reduce the processing time and larger angles are used later if a higher image quality is desired. Angles between $1^{\circ}$ and $6^{\circ}$ were used in this study. The beam entry diameter also affects the processed image. SAFT assumes a synthetic aperture with a point source at the beginning, expanding in the general shape of a cone. Since the aperture is small at the near surface, the number of off-center A-scans used during processing is also small. In order to take advantage of the spatial averaging inherent in SAFT processing, the operator typically enters a value equal to one-half the transducer element diameter. The effect on the synthetic aperture used in the processing is to create a cylinder with a diameter equal to the beam entry diameter parameter that would extend into the material until it intersects the normal aperture cone.

Material properties are also very important to the processing scheme. In the case of the knuckle wall, the material type, wall thickness and acoustic properties are all very well known to a high degree of accuracy. Sampling information includes parameters that are 
related to the temporal sampling (digitization) of the waveform as well as the use of linear averaging for reduction of electronic (white) noise. In order to achieve sufficient sampling, a sample rate of $25 \mathrm{MHz}$ was employed. At an examination frequency of 3.5 $\mathrm{MHz}$, this corresponded to a digitization rate of approximately 7 points per cycle. Due to the attenuative effects of long path lengths and beam divergence, the receive amplifier was required to operate at higher levels than anticipated. At 54-60 decibels $(\mathrm{dB})$ gain, the amplifier introduced noise that was reduced by implementing linear averaging. The step increment in both the $\mathrm{X}$ and $\mathrm{Y}$ axes affects the quality of the processed image. Typically, increment steps of $\lambda / 2$ (where $\lambda$ is the wavelength) are desired; however, in order to reduce file sizes to more manageable levels and decrease the processing times, slightly larger increment step sizes were used. In this study, the wavelength in the material was $0.91 \mathrm{~mm}$, and the incremental step size in both axes was nominally kept at $1.27 \mathrm{~mm}$. Finally, selection of a threshold value for processing can affect the resultant processed file. If a threshold value is selected, and the amplitude of any elementary data element being processed is below this threshold value, then no off-center A-scans will be summed. In this study, a threshold value of $-20 \mathrm{~dB}$ (corresponding to $10 \%$ of the maximum value) was used in order to increase processing speed; however, the threshold value was lowered to $0 \mathrm{~dB}$ later on in the study to increase image quality in hopes of detecting tip-diffracted signals.

\section{SAFT/T-SAFT Evaluation}

\subsection{Flat Plate Analysis}

\subsubsection{Flat Plate Simulation Using IMAGINE3D Software}

A software package to simulate what the sound waves were doing within the test specimens was acquired from UTEX Scientific Instruments Inc. The software package, IMAGINE3D, is a three-dimensional raytracing program. The computer program uses three-dimensional graphics that can be rotated to any viewing angle to fully simulate ultrasonic inspections. Utilizing this software allowed PNNL researchers to predict the most appropriate transducers to use for the inspections as well as help understand the multiple $\mathrm{V}$ path signals that were acquired over the long distances.

Figure 4.1 is a computer simulation of the sound field as it propagates in the flat plate, in the direction of the $5.1 \mathrm{~mm}$ deep reflector, in IMAGINE3D. PNNL has chosen a $70^{\circ}$ shear beam to interrogate the plate.

Figure 4.1 IMAGINE3D Simulation of Flat Plate Inspection Using $70^{\circ}$ Shear Waves 


\subsubsection{Flat Plate Geometry and Reflectors}

The first step in finding a viable solution to the examination of the knuckle region is to look at a flat plate configuration. Initial tests were conducted on a $610 \mathrm{~mm}$ wide by 1219 $\mathrm{mm}$ long carbon steel plate that was $25.4 \mathrm{~mm}$ thick. The idea is to evaluate the ultrasonic

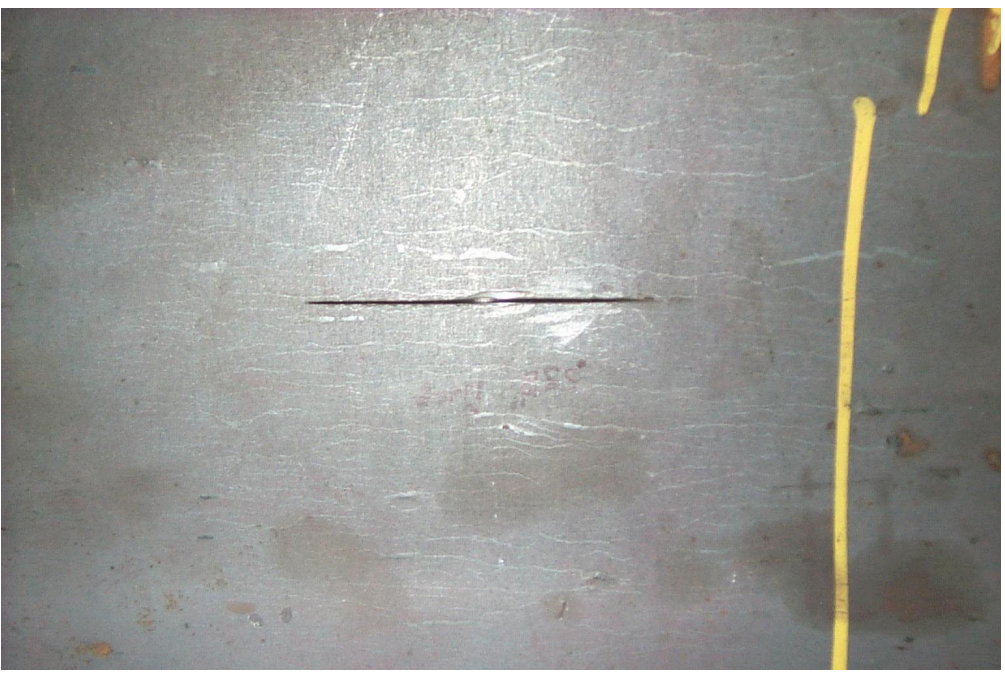
signals returned from the end of the plate at a large distance, i.e. $\sim 1168 \mathrm{~mm}$. These distances approximate the lengths needed for the examination of the knuckle. Ultrasonic scanning was performed on the plate initially without any artificial reflectors to form a test basis. Once the basis data had been acquired, sawcut reflectors (Figure 4.2) were placed in the plate near one end.

Figure 4.2 Sawcut - 12.7-mm Deep by 71.1-mm Long

The sawcuts were oriented parallel to the end of the plate, approximately $102 \mathrm{~mm}$ from the end and on the opposite side of the plate from where the scanning transducer was located. The depths of sawcuts were $5.1-\mathrm{mm}$ deep by 40.6-mm long and 12.7$\mathrm{mm}$ deep by $71.1-\mathrm{mm}$ long. Figure 4.3 is a drawing showing the plate configuration.

\subsubsection{Data Acquisition Configuration}

The flat plate was set on a mobile platform whereby it could be positioned under the scanning system. The pulse echo transducer was situated on the plate approximately $1168 \mathrm{~mm}$ from the end of plate where the sawcuts were located. Figure 4.4

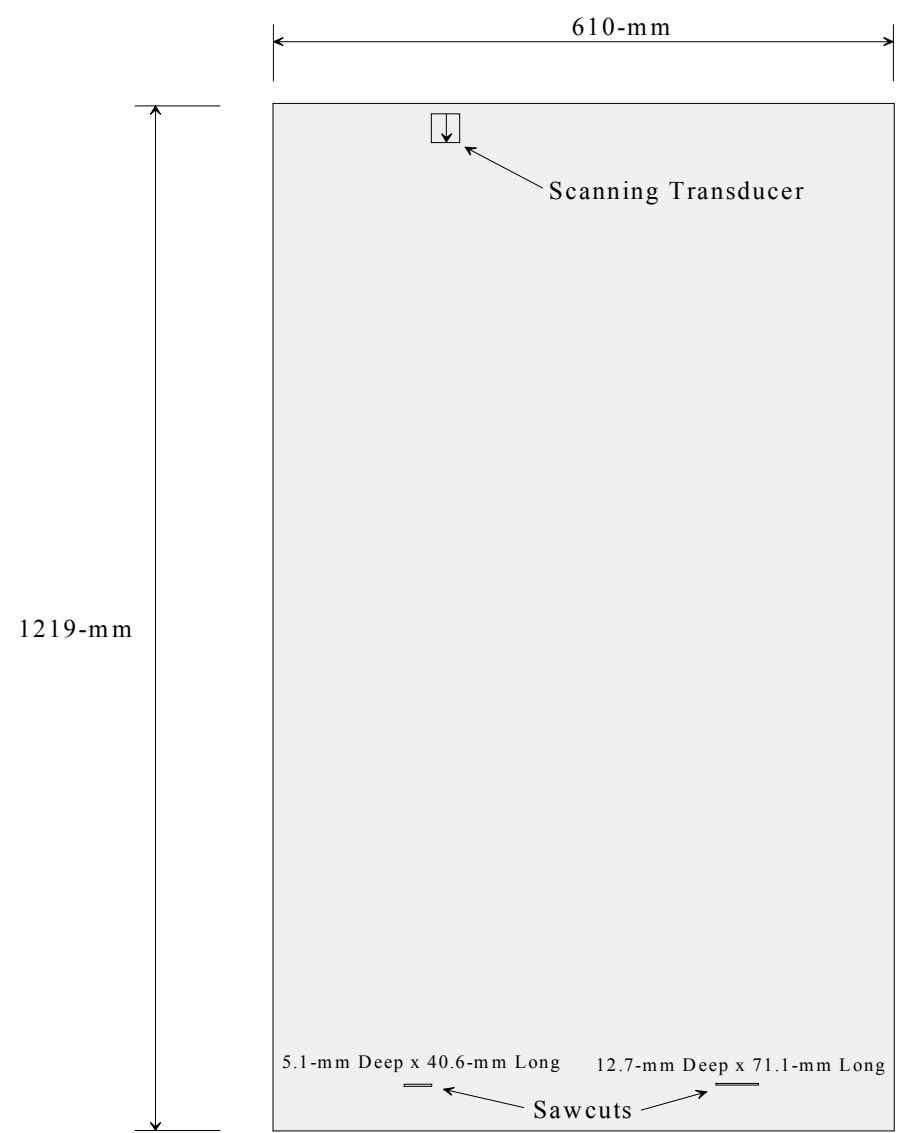

Figure 4.3 Flat Plate Configuration 
shows the data acquisition configuration. A bead of silicon rubber material was used to create a dam for containment of the couplant. Initially, mineral oil was used as the couplant; however, due to suction effects common with larger wedges and lower

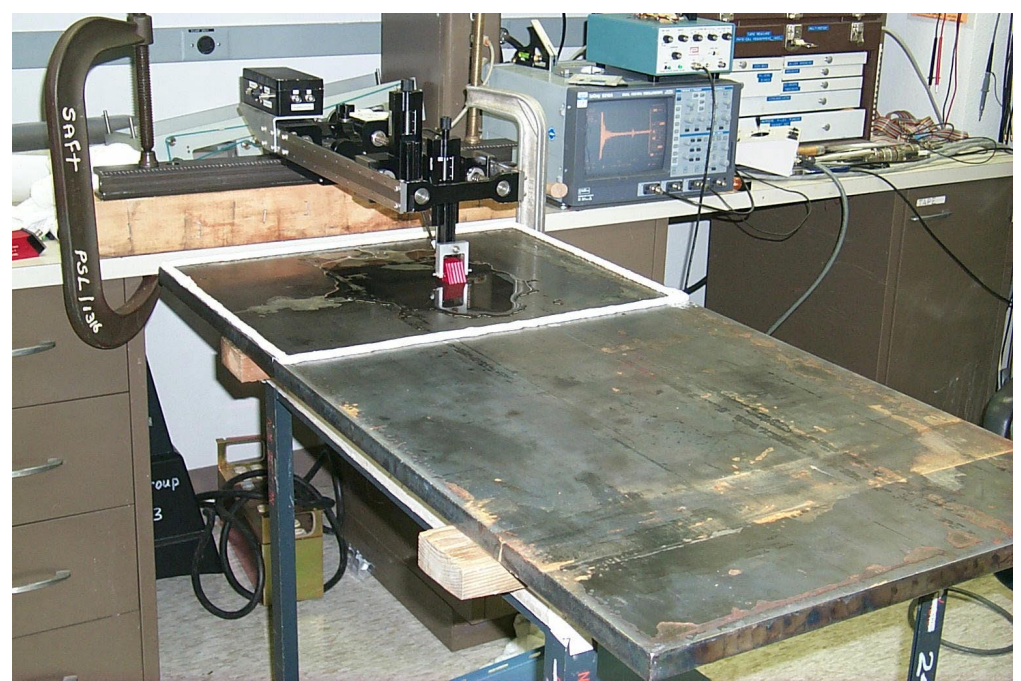

amplitude signal responses as a result of the mineral oil being swept away from the transducer face, water was used in place of the oil. Water proved to work much better, and with proper leveling of the flat plate, the silicon rubber bead was removed and the water would pool around the transducer, eliminating the requirement for a containment mechanism.

Figure 4.4 Flat Plate Test Configuration

The SAFT-UT inspection system is an automated, computerized UT imaging system. The system is a field-tested system, consisting of several components. The pulser produces high-voltage, short-duration, square-wave-pulses that excite the transducer. The receiver conditions and amplifies the received UT response signals with an amplification range of 0-60 dB. The received signal responses (echoes) were initially amplified and then highpass filtered in order to allow suitable amplification while reducing extraneous low-frequency noise components under 300 kilohertz $(\mathrm{kHz})$.

A Pentium-based PC containing an analog-to-digital (A/D) card and motor driver cards provides a platform for both SAFT data processing and storage of UT data files. The $\mathrm{A} / \mathrm{D}$ converter was used to acquire (digitize) data and initialize a trigger output to the motor controller and pulser-receiver which, in turn, was used to sync other instrumentation. This system contains a re-writable CD-ROM drive to store digital data. The PC is also a complete data display and analysis system for displaying the SAFT-UT processed data (A-scans, B-scans, and C-scans). The data acquisition electronics are coupled to an automated pipe scanner (with a flat scanning track and spring-loaded gimbal fixture) that is used for accurate and smooth continuous scanning of the search unit through a specified number of points in the X-Y plane, while maintaining low noise conditions.

All signals (excitation pulses and received signal responses) were monitored using a LeCroy digital oscilloscope, providing the capability to view the trigger and sync pulses, the excitation pulse, and the received signal response just prior to digitization, simultaneously. The LeCroy oscilloscope also provided linear averaging, and the capability to analyze the frequency characteristics of the A-scan data. 
A linear averaging scheme was implemented with the rf ultrasonic A-scan data being averaged up to eight times per scanning position, in order to minimize the effects of motor noise and random electronic noise, and increase the unprocessed SNR. The limiting factor associated with implementing higher averaging rates was scan speed.

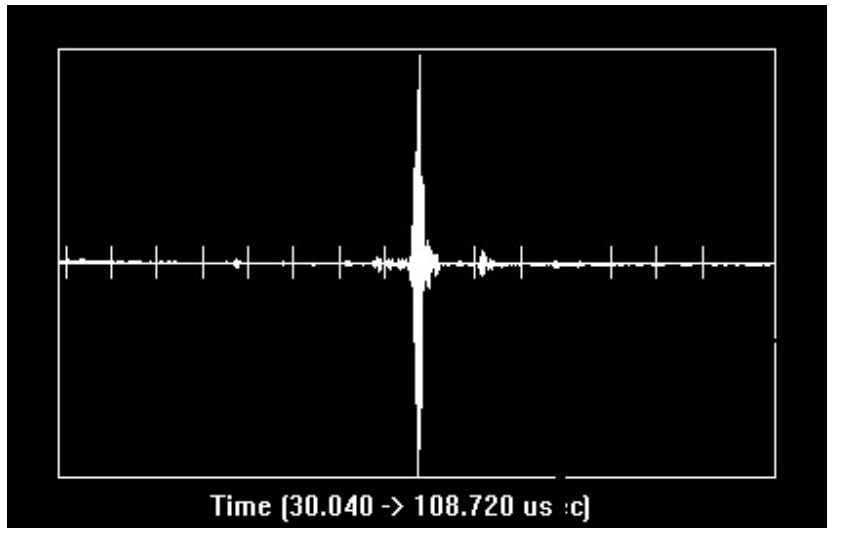

a) $1 / 2 \mathrm{~V}$

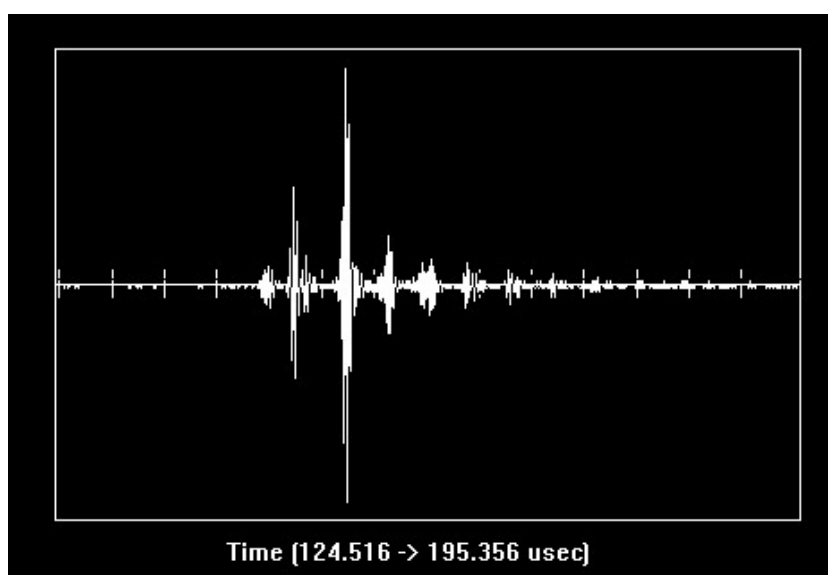

b) $3 / 2 \mathrm{~V}$

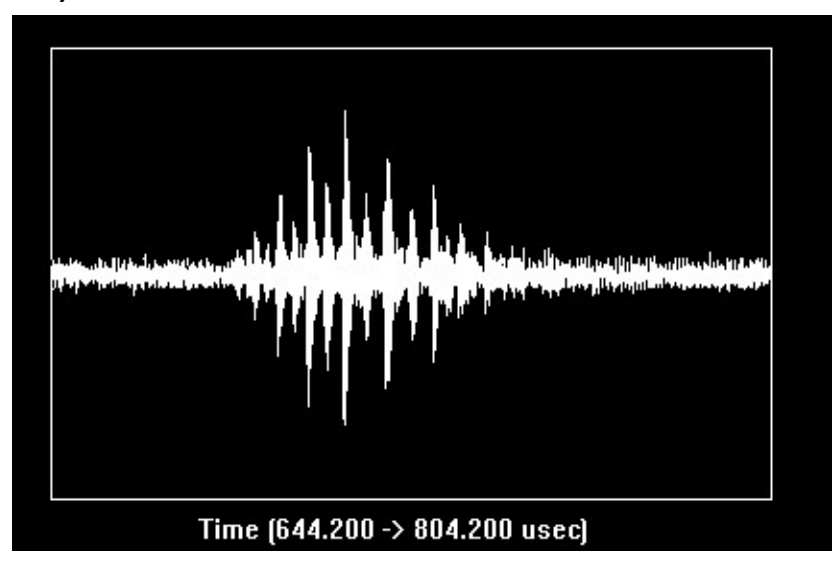

c) End of Plate

Figure 4.5 Evolution of Wave Packet as a Function of Path Length from End of Plate

\subsubsection{Examination of End-of- Plate}

In order to fully understand the various conditions that could affect sound field propagation in the knuckle region and beyond, the initial effort began with an examination of the signal responses from the end of a flat plate. The corner-trap response from the end-of-plate was recorded as a function of wave mode (longitudinal vs. shear), examination frequency $(500 \mathrm{kHz}$ to $5.0 \mathrm{MHz})$, incident angle $\left(60^{\circ}\right.$ and greater), and path length, ranging from $1 / 2 \mathrm{~V}$ to over 7 full$\mathrm{V}$ paths (corresponding to over 1118-mm of surface path). In this study, attenuation, mode conversions, beam divergence, coupling issues, weld effects, and reflector geometry and dimensions all played key roles in shaping the process to determine the optimal scheme for sound propagation. This effort focused on evaluating wedgemounted, contact transducers coupled to the outer wall surface where the examinations targeted inner wall surface connected reflectors. Examination results showed that $70^{\circ}$ incident shear waves at $3.5 \mathrm{MHz}$ provided the best resolution characteristics while maintaining sufficient acoustic energy over the extended path lengths. 
Figure 4.5 depicts the A-scan signal responses and how they evolve as a function of path length from the end of the plate. As the transducer is scanned at longer distances from the end-of-plate, the divergence of the beam coupled with off-angle signal responses from the corner trap provide a "packet" of signal responses. This set of multiple signal indications corresponds to the received $70^{\circ}$ primary ray and received angles about the primary ray. The data shows a consistent decrease in signal-to-noise levels as the transducer is scanned at greater distances from the plate end. This same phenomena was exhibited with signal "packet" responses from the sawcuts examined in this study as well.
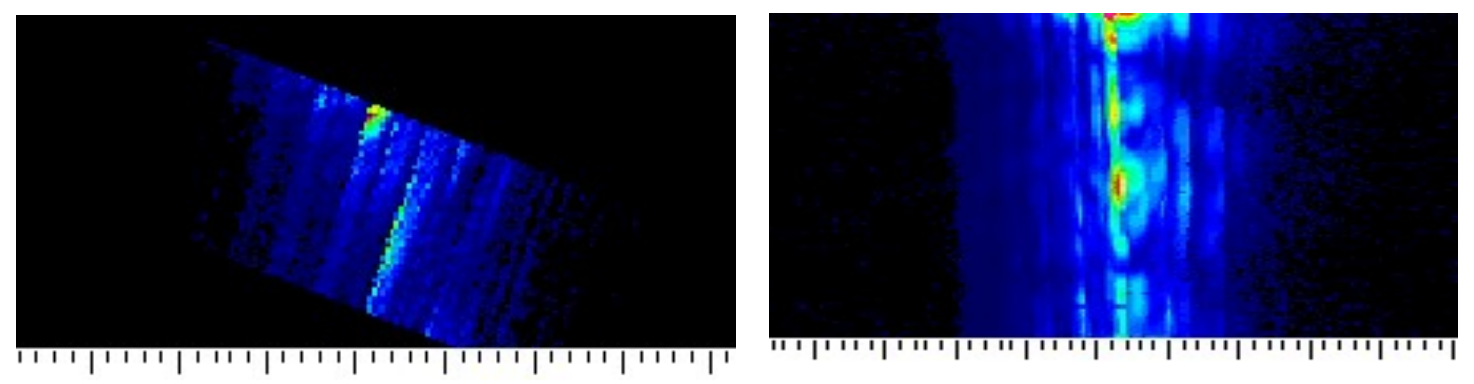

Figure 4.6 SAFT-Processed B-Scan Side View (Left) and C-Scan Top View (Right) of End of Plate

The raw ultrasonic signal responses from the end-of-plate were of high amplitude and consistently detected. SAFT processing provided a more focused image of the "packets" and corrected for "smearing" of the resultant data. Detection of the end-of-plate corner trap was a necessary first step in understanding the received ultrasonic signal responses and determining the propagation effects of the sound field over such long distances. Figure 4.6 illustrates the resultant SAFT-processed B- and C-scan images of the end-ofplate over the maximum distance scanned in this study (approximately $1143 \mathrm{~mm}$ ). The left image in Figure 4.6 is a projected B-Scan at $70^{\circ}$. The elongated higher amplitude signal shown symmetrically in the center of the scan is the end of plate signal. The right image in Figure 4.6 is a C-Scan of that end of plate signal. 


\subsubsection{Examination of Sawcuts}

The next step in the study was to introduce physical reflectors (sawcuts) into the flat plate specimen and evaluate the performance of the inspection technique for detection and localization of reflectors as a function of path length and reflector dimensions. As expected, the signal responses from the corner-trap of the sawcuts exhibited the "packet" phenomena as the transducer was scanned at greater path lengths from the reflector. Figure 4.7 depicts the Ascan signal response "packet" from both sawcuts at the maximum allowable distance of 1067$\mathrm{mm}$. The data illustrates the loss of signal amplitude from the end-ofplate corner trap response for the case of the $12.7-\mathrm{mm}$ deep sawcut. This was expected, as a larger portion of the sound field is reflected back to the transducer from a sawcut that is $50 \%$ through-wall in comparison to a $20 \%$ through-wall sawcut (the 5.1-mm

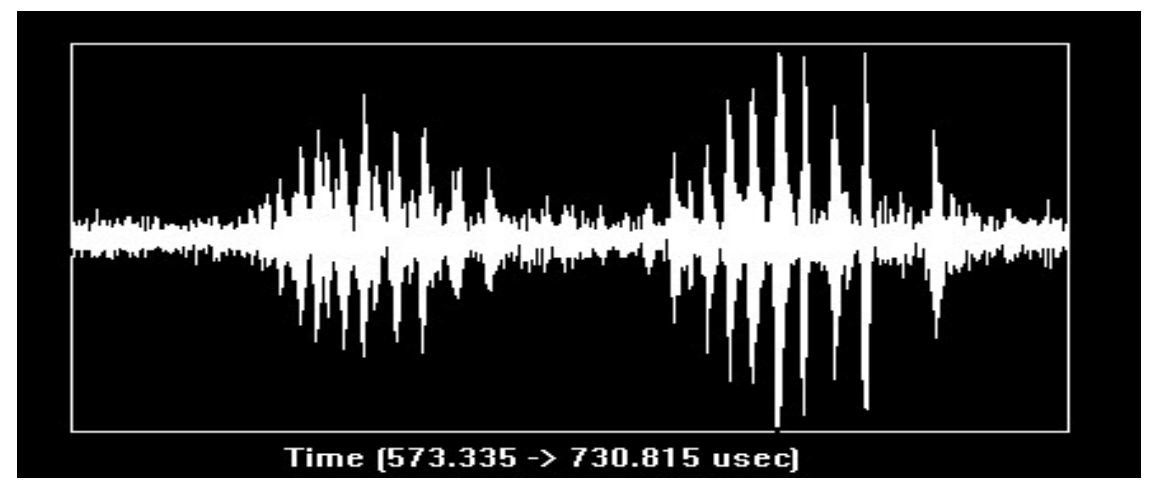

a) Signal Response from 5.1-mm deep Sawcut and End of Plate

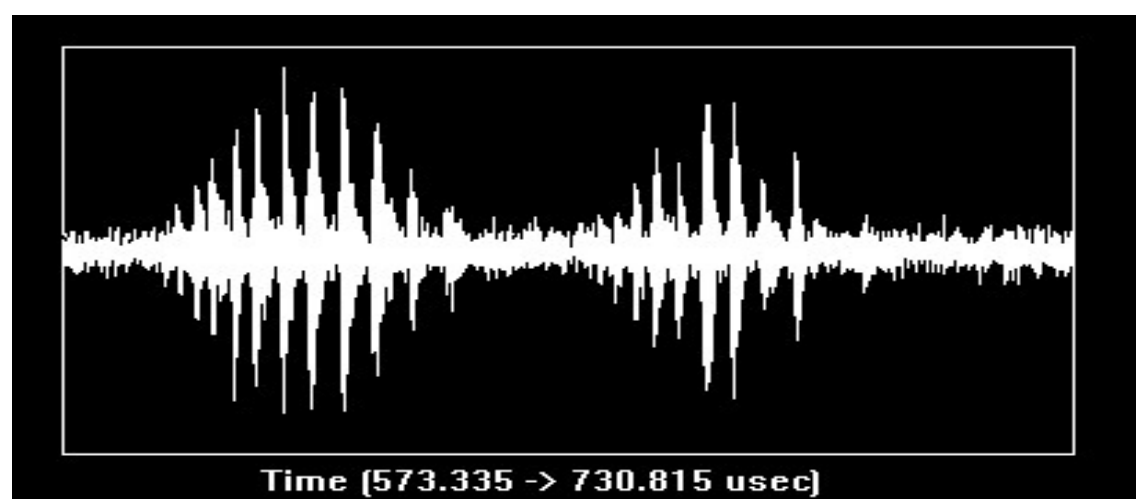

b) Signal Response from 12.7-mm deep Sawcut and End of Plate

Figure 4.7 A-Scans of Small and Large Sawcuts deep sawcut).

The dynamic range of the signal responses was maximized by peaking the signal amplitudes at the correct geometrical distance from the reflector to correspond with a specified $1 / 2 \mathrm{~V}$ path or multiple $1 / 2 \mathrm{~V}$ path. In this manner, the signal response from the end-of-plate was identified and saturated, and system gain was near maximum (in the range of 54-60 dB). The signal-to-noise ratio for the signal responses from the sawcuts was lower than that from the end-of-plate, as expected. However, the data showed that the technique was capable of detection and localization (relative to their positions from 
the end-of-plate) of both sawcuts (12.7-mm and 5.1-mm deep) at the maximum distance allowed on the flat plate (approximately $1067 \mathrm{~mm}$ ). Figure 4.8 illustrates the SAFT processed B-scan and C-scan images of the 5.1-mm deep sawcut in the flat plate. The large tic marks are $25.4 \mathrm{~mm}$ apart. On the left image, the bright signal seen on the right hand side is the end of plate signal. The other image is the 5.1-mm deep sawcut approximately $102 \mathrm{~mm}$ from the end of plate.
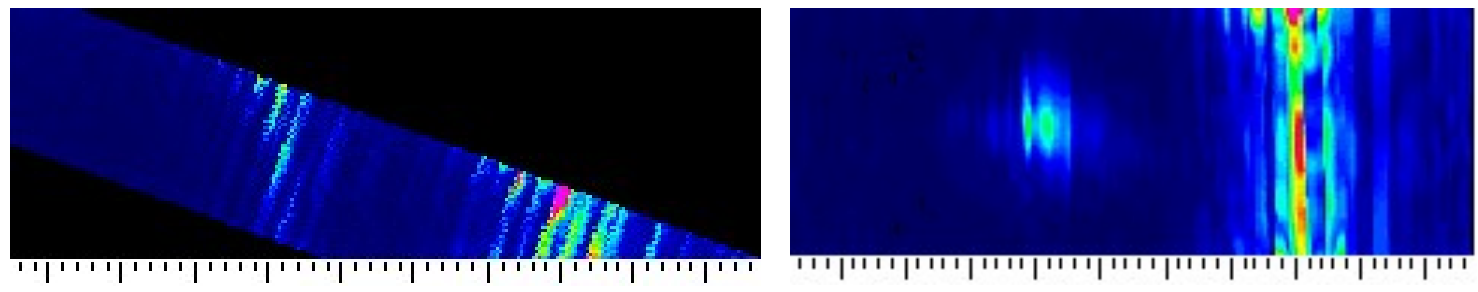

Figure 4.8 SAFT-Processed B-Scan Side View (Left) and C-Scan Top View (Right) of 5.1-mm Sawcut

The data indicates that the technique is quite capable of detection and localization of sawcut reflectors 5.1-mm deep and greater, in the 25.4-mm thick carbon steel plate from distances up to $1067 \mathrm{~mm}$ from the transducer. The examination frequency allows for suitable resolution over this distance.

\subsubsection{Weld Effects}

Examination of the knuckle region may include one or more welds. All tanks include a circumferential weld located on the vertical portion of the tank. Some tanks also include a circumferential weld located approximately $305 \mathrm{~mm}$ under the tank just above the insulating concrete pad. All tanks include a transition weld located approximately 1219 mm under the tank.

Weld effects were investigated using the original flat plate with the sawcuts near the end of plate. The original flat plate had been scanned to provide basis data from the end of plate and the 5.1-mm sawcut. The flat plate was then cut, weld prepped, and rewelded using a similar welding technique as that used on the waste tanks. Figure 4.9 shows the welded configuration and scanning technique.

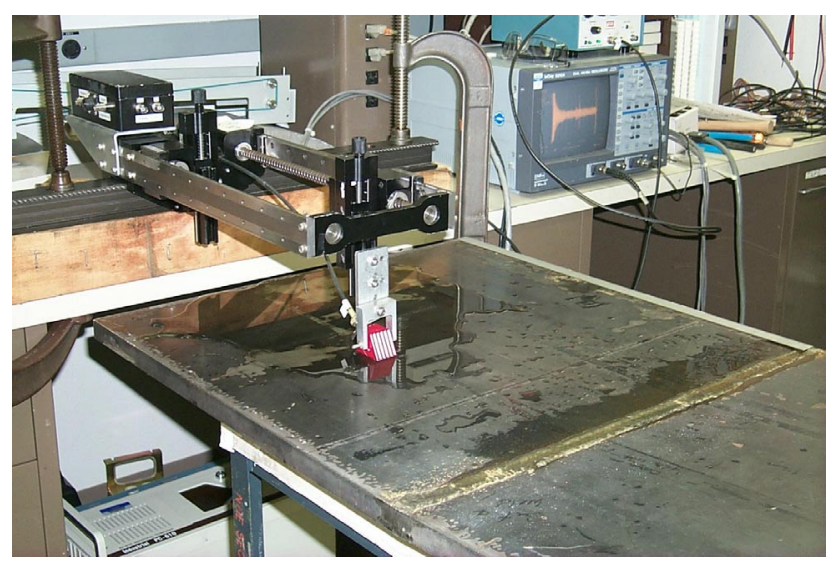

Figure 4.9 Scanning of Flat Plate with Weld
Sound was propagated from the blank end, through the weld, to examine the 5.1-mm sawcut. As shown in Figure 4.10 , the end of plate is the high amplitude signal on the right side of each picture. The sawcut is shown approximately $102 \mathrm{~mm}$ in front of the end of plate signal in each picture. The image on the left was processed from data acquired with no weld in the plate. The image on the right corresponds to the welded condition. 
The current status of the SAFT algorithm does not allow for additional focusing of the signal, therefore the sawcut looks smeared. The weld does cause some further scattering of the return signal as well as an amplitude degradation; however, the SNR is still very good. The dynamic range of the two images are at different levels.

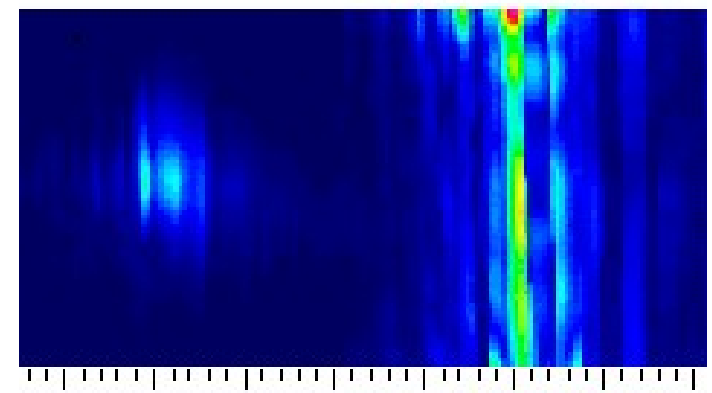

a) Scan with no Weld

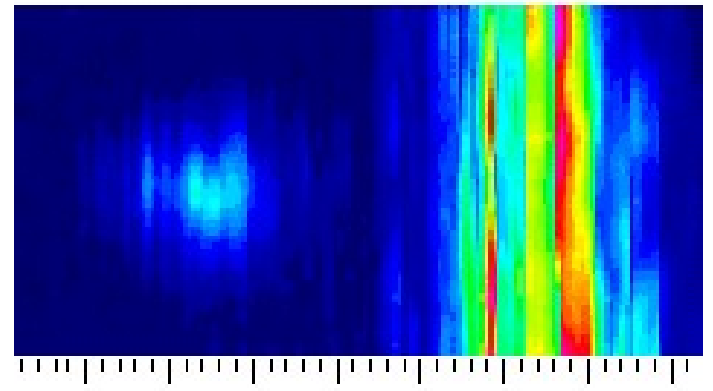

b) Scan with Weld

Figure 4.10 SAFT-Processed C-Scan Comparison of 5.1-mm Sawcut Through Weld

\subsubsection{Summary of Flat Plate Examinations}

The results clearly indicate a successful demonstration of the $70^{\circ}$ shear wave inspection technique for detection and localization of reflectors in a 25.4-mm thick carbon steel flat plate for distances ranging from approximately $889 \mathrm{~mm}$ to $1067 \mathrm{~mm}$. Although signal response amplitudes vary with the addition of weldment geometry between the transmitting transducer and machined reflectors, the enhancements provided by SAFT processing provide an effective means to compensate for these effects and appear to be very promising for the development of an optimized inspection protocol for the knuckle region and beyond. The analysis of data acquired from examinations in the laboratory show that the inspection technique is capable of detecting and localizing machined reflectors, over very long path lengths and through a weld, with through wall sizes of the order of 5.1-mm deep.

\subsection{Mock-up Analysis}

\subsubsection{Mock-up Simulation Using IMAGINE3D Software}

The simulation software described in Section 4.1.1 provided an effective platform for assessing various inspection parameters that might affect the inspection approach. The simulations supported this effort by expediting a more optimized development. The simulation process included visualizing the two-dimensional sound field and its path as a function of incident angle, wave mode, frequency, wedge and coupling characteristics, reflector geometry and dimensions and material properties. This allowed PNNL staff to more efficiently diagnose potential inspection problems prior to beginning the experimental trials that might have otherwise increased programmatic costs and slowed progress. Various transducer configurations were examined and of primary importance was understanding the effects of the knuckle region geometry on the $70^{\circ}$ incident shear 
wave acoustic field. Potential mode conversions were monitored as a function of the position and relative coherence of the signal responses from the resultant insonified volume. This tool was invaluable in guiding the effort down the most effective path on the shortest time schedule. Figure 4.11 provides an IMAGINE3D simulation of the

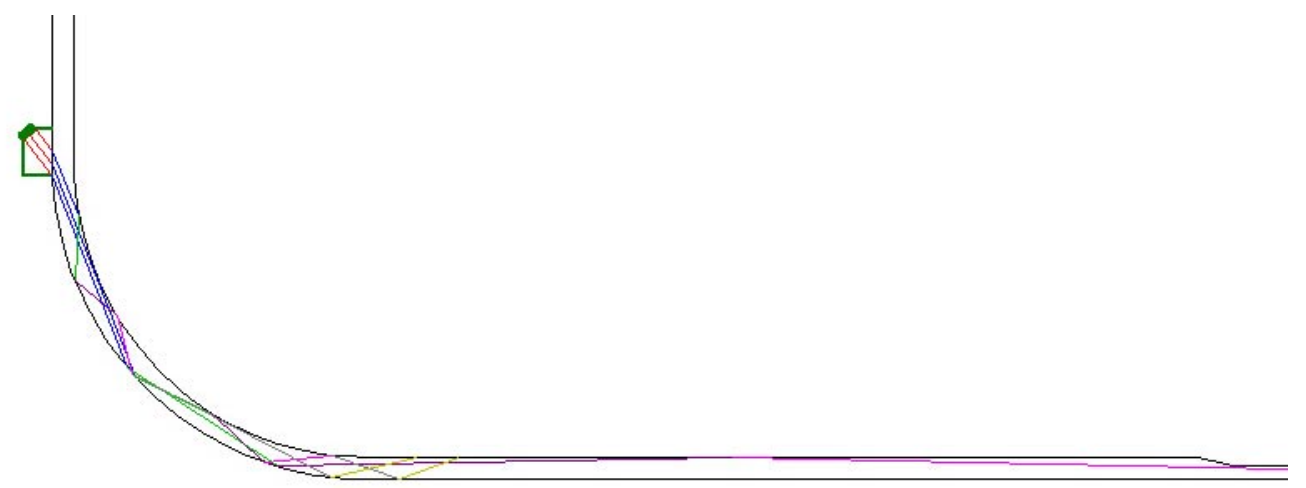

Figure 4.11 IMAGINE3D Simulation of Knuckle Region

knuckle region and provides insight into wave propagation in this region. At certain positions above the knuckle the waveform changes. Some positions exhibit very symmetrical bounces with equal path lengths, while other positions exhibit short and long path lengths. These correlate to time of flight changes, which can affect the SAFT processing parameters.

\subsubsection{Mock-up Geometry and Reflectors}

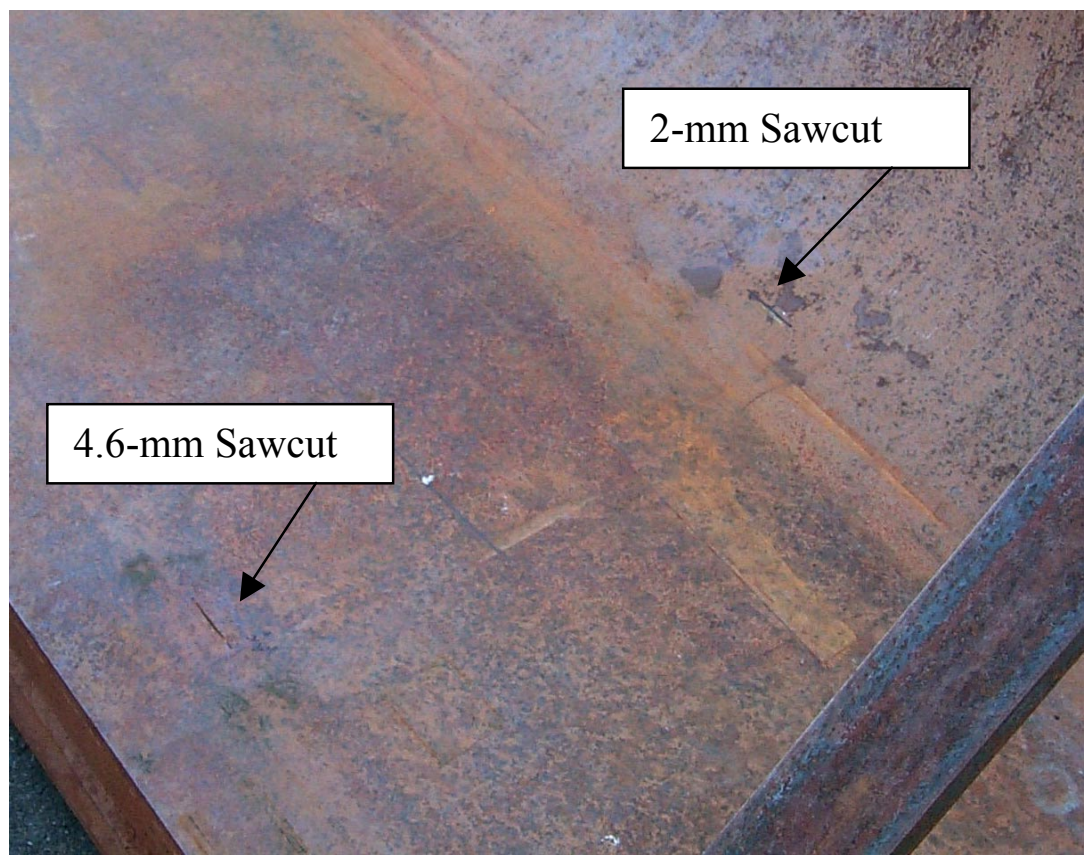

Once the baseline data was completed on the flat plate case, the scanning system was moved out to the fullscale mockup of the knuckle region. Initial scanning of the knuckle and beyond to the end of the knuckle plate was completed. The next step included machining a sawcut into the area approximately $102 \mathrm{~mm}$ from the end of the knuckle plate.

Figure 4.12 Mockup Knuckle Showing Sawcuts 
This is approximately the same distance that was inspected in the flat plate case and would allow for an assessment of the effects of the knuckle on the inspection process. The sawcut was approximately $4.6 \mathrm{~mm}$ deep and $39.4 \mathrm{~mm}$ long. The depth of the sawcut is approximately the same as the minimum crack depth requirement for detection and sizing as required in Table 2.1. A second sawcut was placed into the knuckle at the predicted highest stress region. This sawcut was $2 \mathrm{~mm}$ deep and $27.9 \mathrm{~mm}$ long. Figure 4.12 shows the sawcut locations in the knuckle and near the end of the knuckle plate. The curved portion of the knuckle is located on the right side of Figure 4.12 and the end of the knuckle plate is visible in the left corner. Refer to Figure 4.13, which provides another view of the knuckle region. Sawcuts are on the inside diameter in this figure.

\subsubsection{Data Acquisition Configuration}

The data acquisition system configuration was identical to that used on the examination of the flat plate in the laboratory. The only significant difference was in the mounting of the automated pipe scanner to the mock-up wall. A long rectangular extruded aluminum bar with grooved sides was c-clamped to the entire length of the mock-up and used as a support structure to bolt the flat scanner track against. This provided a means to attach the pipe scanner and properly mount transducers in either a pulse-echo or tandem scanning mode oriented on the outer tank wall and directed towards the knuckle region. Figure 4.13 illustrates the scanning system configuration on the mock-up. All other system components remained identical to that described in Section 4.1.3.

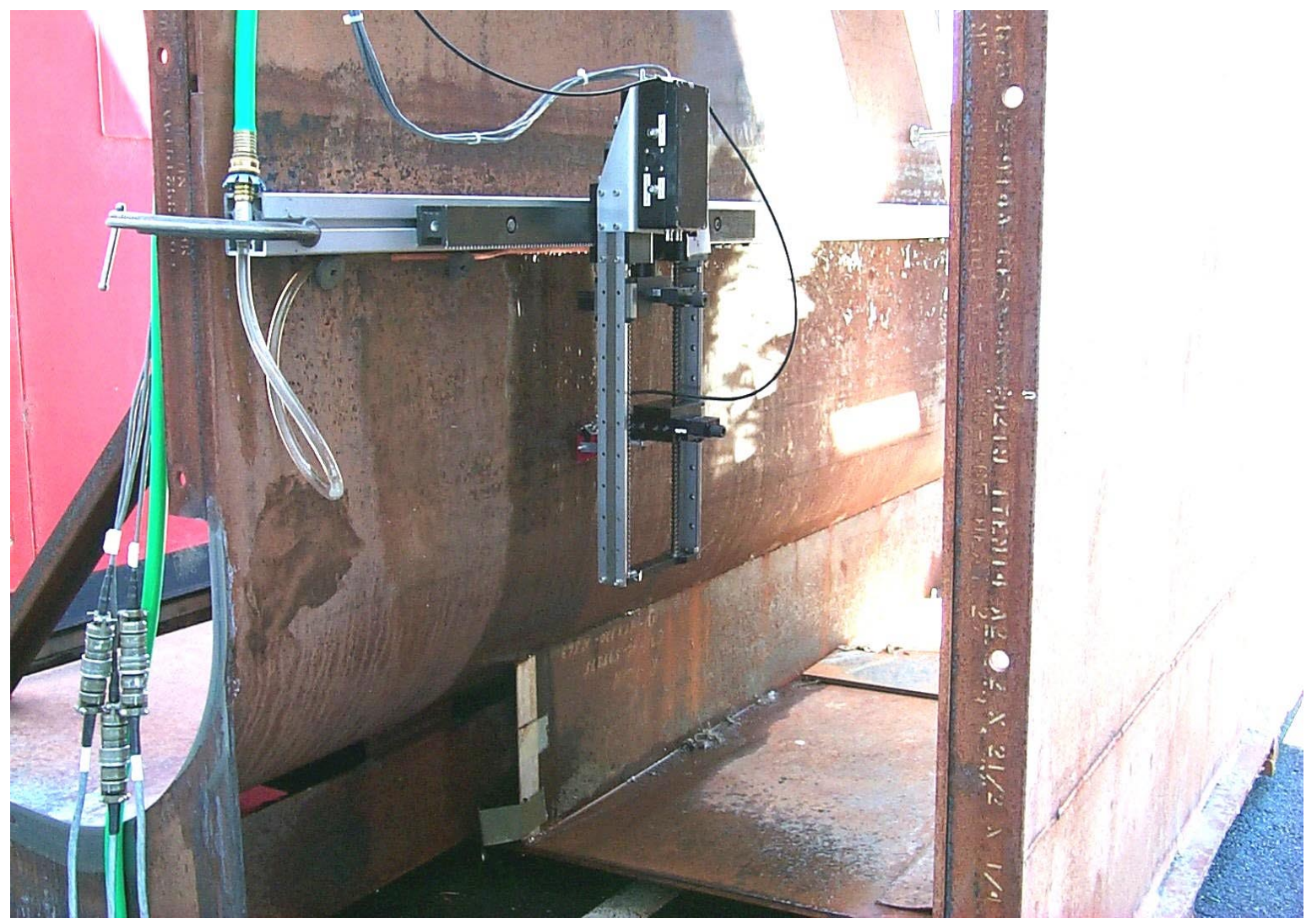

Figure 4.13 Scanning System on Mockup 
A copper tube with multiple holes drilled into it was located at the base of the extruded aluminum bar. This copper tube was held in place by magnets and was connected to a water supply line. The supply line was controlled using a valve and plastic tubing, and was configured to provide a constant flow of water coupling down the outer wall surface as the transducer scanned the knuckle region. This coupling platform worked very well for the mock-up examinations.

The configuration for dual transducer scanning required that special fixturing and gimbals be fabricated in order to scan two identical transducers in side-by-side fashion (for tandem mode scanning) in very close proximity to one another. This configuration was only implemented on the mock-up. The photograph shown in Figure 4.14 depicts the tandem mode scanning configuration, where one transducer acts solely as a transmitter and the other as a receiver.

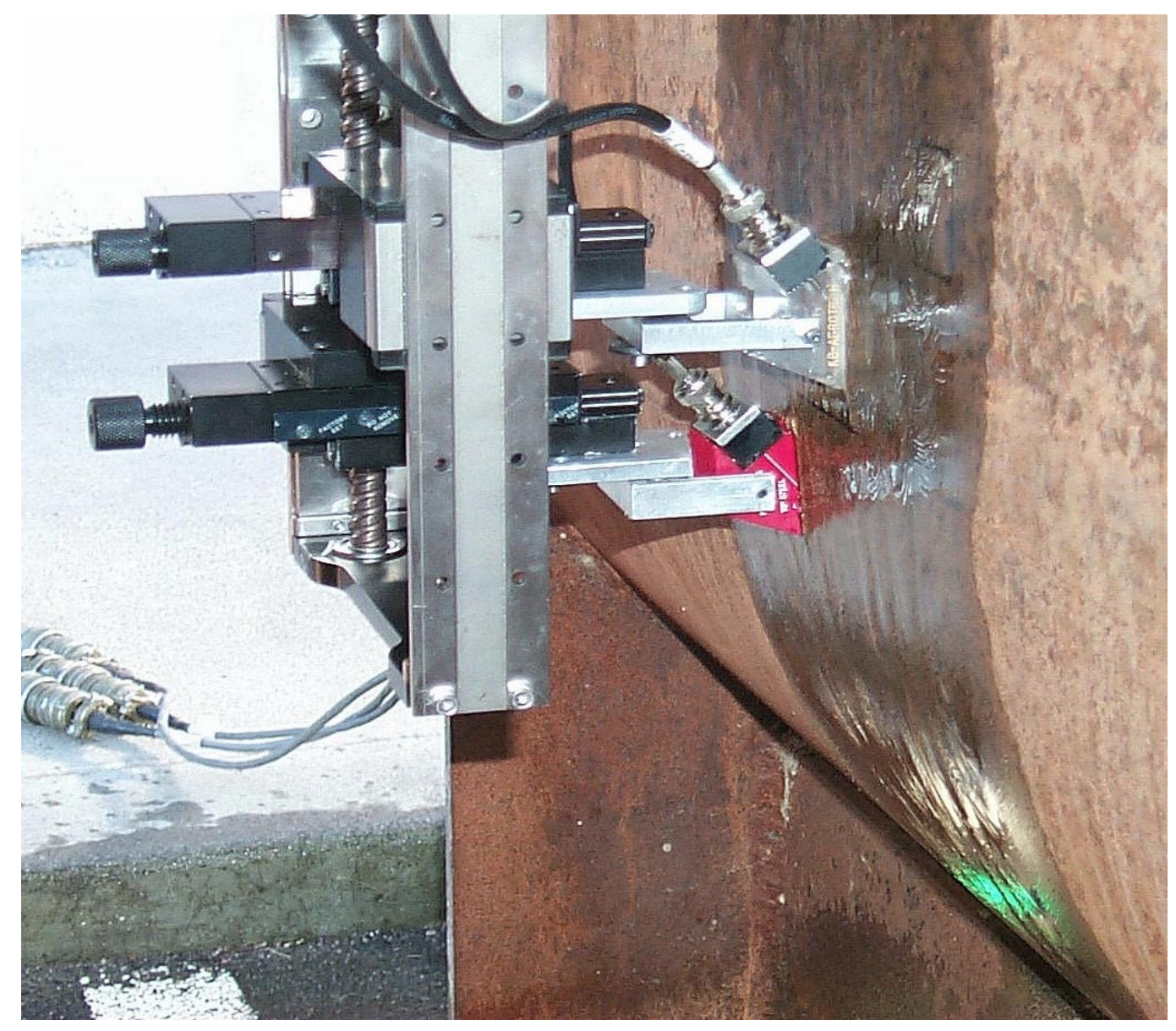

Figure 4.14 T-SAFT Scanning Configuration on Mockup 


\subsubsection{Examination of End-of-Knuckle on Mock-up}

The evaluation protocol was identical to that conducted on the flat plate, with the addition of performing some additional tandem mode scans for acquisition of initial sizing information. In order to fully understand the effects of the knuckle region on sound field propagation, this phase of the effort began with an examination of the signal responses from the end of the knuckle, at a distance of approximately $1092 \mathrm{~mm}$. The corner-trap response from the end-of-knuckle again exhibited a "packet" of signal response peaks, due to receiving simultaneous off-angle A-scan responses from the corner-trap.

However, the symmetry of the packet was distorted due the geometrical effects of the wavefront as it propagates through the knuckle. Figure 4.15 illustrates the comparison between an A-scan "packet" end-of-plate signal response from the flat plate, and an Ascan "packet" end-of-knuckle plate signal response from the mockup.

As in the case of the flat plate, the raw ultrasonic signal responses from the end-ofknuckle were of high amplitude and consistently detected. Again, SAFT processing provided a more focused image of the "packets" and corrected for "smearing" of the
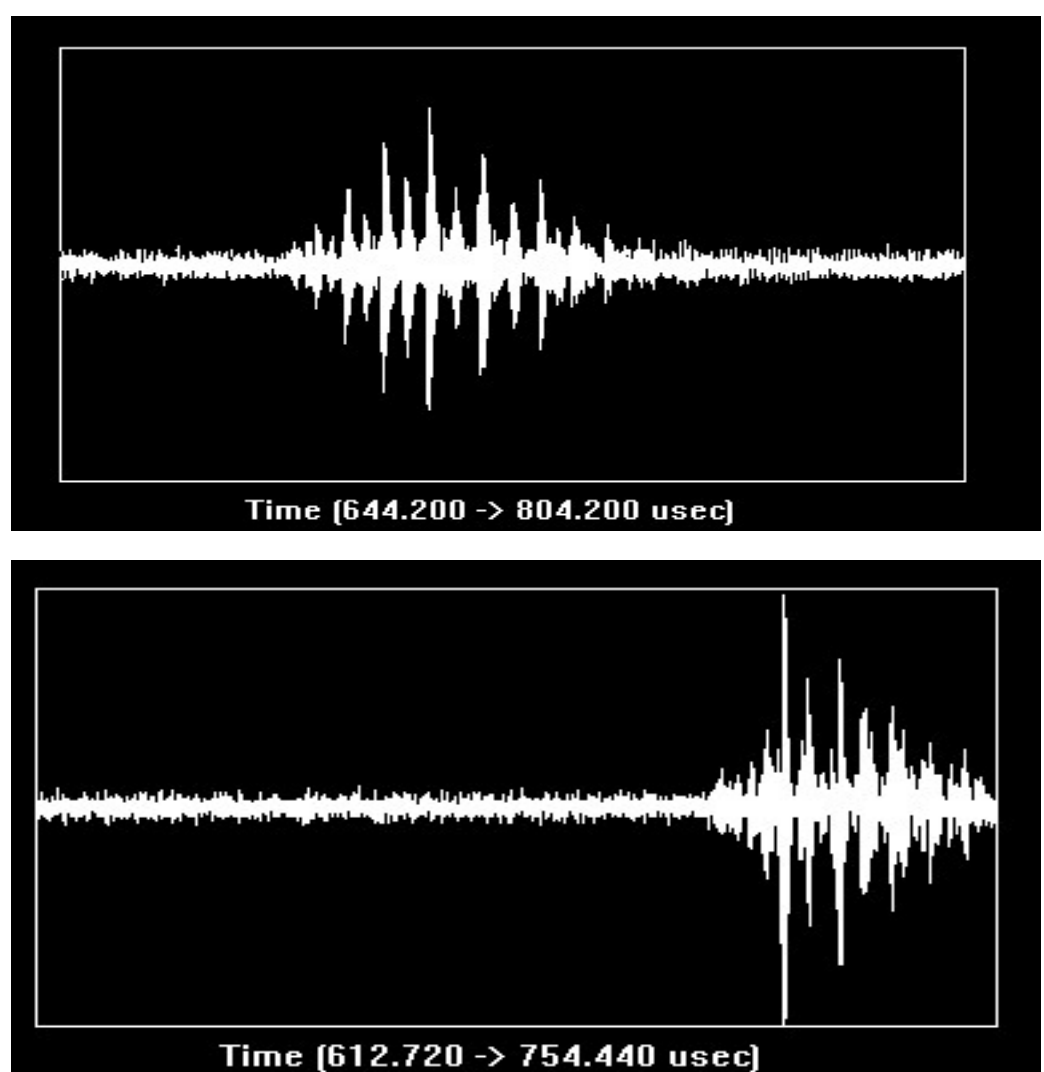

Figure 4.15 Comparison of A-Scan Wave Packets Between

\section{the Flat Plate (top) and the Mockup (Bottom)} resultant data. A large area of essentially blank material (void of any known reflectors) was scanned and marked on the outer surface for future repeat scanning. This scanned area provided baseline data for comparison of noise levels and potential anomalies prior to the addition of machined reflectors (sawcuts) in this region of material. Figure 4.16 illustrates the resultant SAFT-processed B- and C-scan images of the end-of-plate over the maximum distance scanned in this study (approximately $1092 \mathrm{~mm}$ ). 

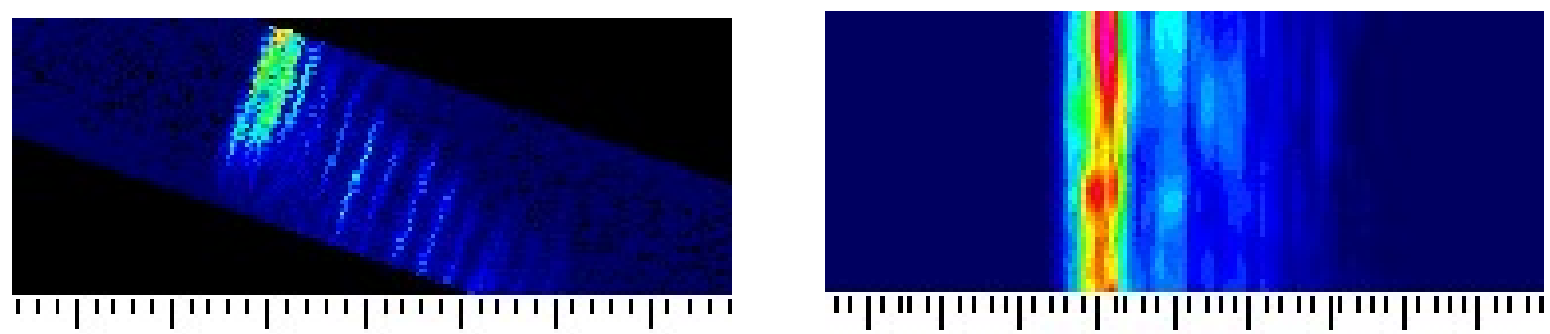

Figure 4.16 SAFT-Processed B-Scan (Left) and C-Scan (Right) Images of the End of Knuckle Plate on Mockup

\subsubsection{Examination of Sawcuts}

Keeping to the same process as that conducted in the laboratory, the PNNL team added a machined reflector (sawcut) located $102 \mathrm{~mm}$ from the end-of-knuckle plate and $4.6 \mathrm{~mm}$ in depth. Again, the performance of the inspection technique was evaluated for detection and localization of this reflector as a function of path length and reflector dimensions. As expected, the signal responses from the corner-trap of the sawcut exhibited the "packet" phenomena as discussed in previous sections. In comparison to the flat plate results, the signal-to-noise ratio was 2-4 dB lower for the mock-up scans; however, the signal responses were relatively strong and consistent with good SNR. Once again, the signal response from the end-of-knuckle was identified and saturated, and system gain was near maximum (in the range of 58-60 dB). At this point, the technique had again proven to be robust, and provided a successful demonstration of the capability to detect and localize the 4.6-mm sawcut near the end-of-knuckle plate mock-up, approximately $965 \mathrm{~mm}$ from the transmitting transducer. Figure 4.17 shows the resultant B-scan and C-scan SAFTprocessed images from the 4.6-mm sawcut.
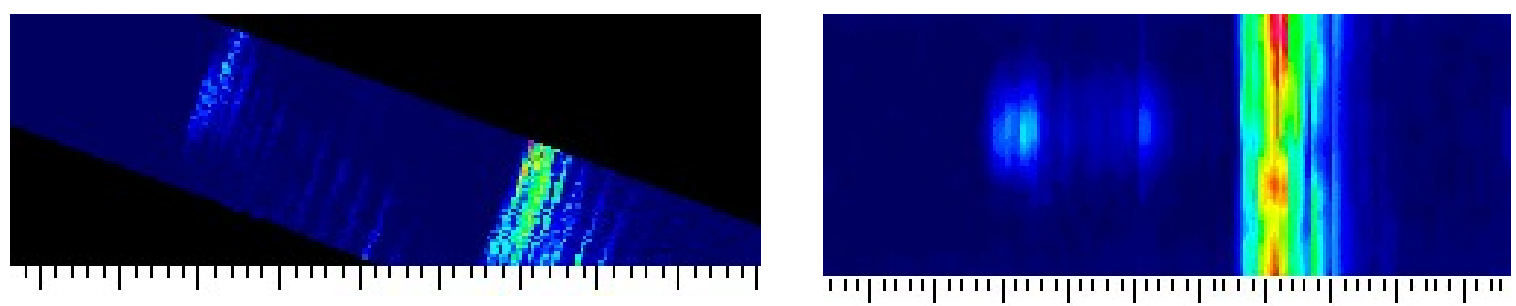

Figure 4.17 SAFT-Processed B-Scan (Left) and C-Scan (Right) Images of 4.6-mm
Sawcut on Mockup

The next step was to introduce a second machined reflector (sawcut) in the knuckle region (knuckle to base transition) located approximately $610 \mathrm{~mm}$ from the end-ofknuckle plate and approximately $483 \mathrm{~mm}$ from the scan start position of the transmitting transducer. This sawcut was fabricated to be only 2-mm deep and was used to examine the effectiveness of detecting reflectors in the knuckle region and relate the performance 
of the inspection technique to the associated effects of sound propagation around the curved region of interest.

Since there were no signal responses from any nearby reflector (i.e., a corner trap from the end-of-knuckle plate), the signal response from the sawcut was used to maximize the dynamic range. The signal response was identified and peaked at a system gain of $50 \mathrm{~dB}$ (10 dB less gain than the previous sawcut), and the time-of-flight curve was used to minimize the digitized window and maximize coverage in the scan axis. The data showed that the technique was capable of detection and localization (relative to the position of the signal response from the transmitter position) and the signal responses were very strong (high SNR) and consistent due to a decreased path length. Figure 4.18 illustrates the SAFT-processed B-scan and C-scan images from the SAFT examination of the 2-mm deep sawcut.
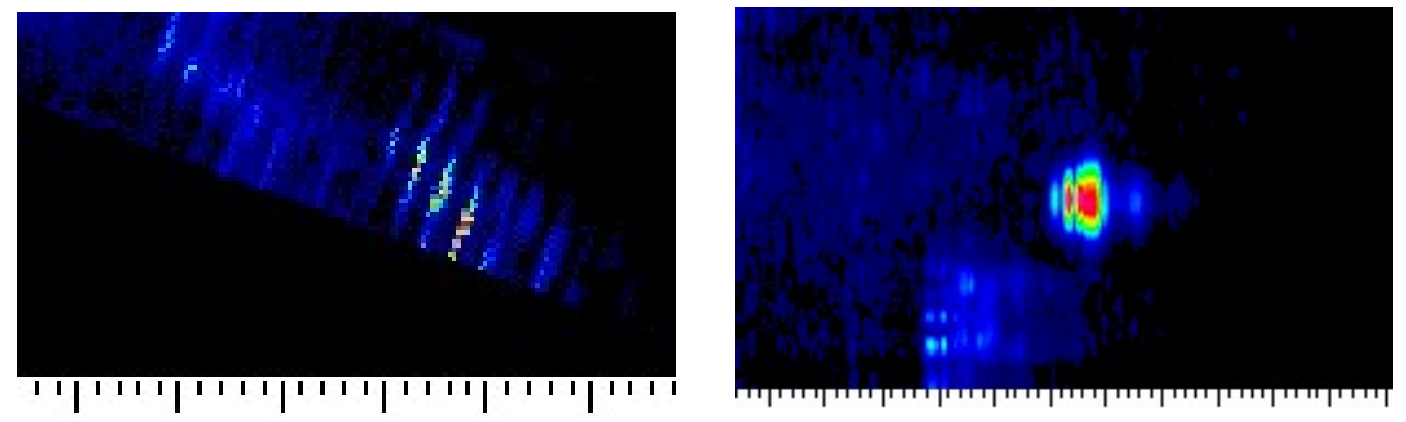
Figure 4.18 SAFT-Processed B-Scan (Left) and C-Scan (Right) Images of 2-mm
Sawcut on Mockup

Finally, in order to make an initial attempt at evaluating the T-SAFT inspection protocol for sizing of reflectors, the system was configured for T-SAFT mode scanning, and a tandem scan of the 4.6-mm deep sawcut (102 mm from the end-of-knuckle plate) was conducted. Since tip diffracted signals are not evident in the pulse-echo data, the implementation of T-SAFT is required for accurate and reliable sizing of defects in the knuckle region and beyond. Presently, the T-SAFT processing software is not optimally configured for processing long path length data; however, the software was "patched" to yield some preliminary results that provided a basis for supporting the use of T-SAFT for flaw sizing. Figure 4.19 shows the pertinent T-SAFT-processed images depicting the
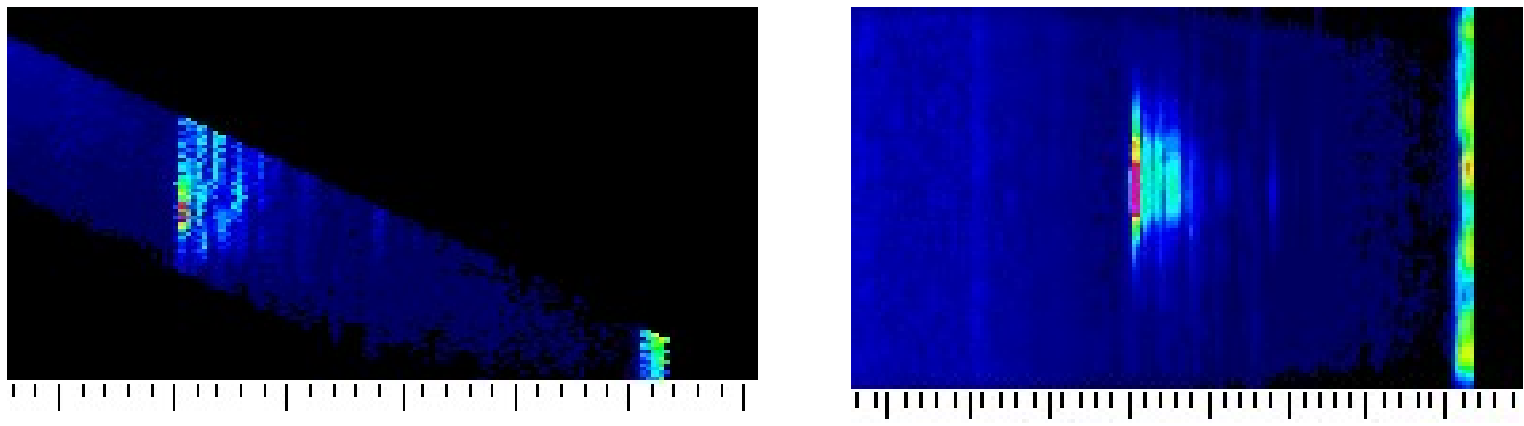

Figure 4.19 T-SAFT-Processed B-Scan (Left) and C-Scan (Right) Images of 4.6-mm Sawcut on Mockup 
sawcut. The images look very similar to those in previous sections with the exception of the high amplitude area. The high amplitude area shown in the center of the C-Scan image can be used for sizing of the defect. This is done in an analysis mode where the software allows you to box out the high amplitude region and perform a $6 \mathrm{~dB}$ drop analysis. From this processed data, the sawcut was sized to be 5.1-mm deep and $29.2 \mathrm{~mm}$ in length. The actual dimensions for the sawcut were 4.6-mm deep and $39.4 \mathrm{~mm}$ in length.

\subsubsection{Weld Effects}

Initially, the details associated with the design and fabrication of the mock-up did not reveal the presence of any welds in or around the knuckle region. In order to verify the existence of one or more welds, (as the mock-up did not visually exhibit any signs of a weld in this region on either inside diameter or outside diameter wall) the edge of the mock-up region where the knuckle begins and ends was machined down and polished, and then chemically etched in order to reveal any weldment geometry that may exist. This effort verified that no welds existed in the mock-up and, therefore, any weld effects coupled with the geometrical effects of the knuckle were not examined in this study.

\subsubsection{Summary of Mock-up Examinations}

The results of the mock-up examinations clearly indicate the successful demonstration of SAFT processing coupled with the $70^{\circ}$ shear wave inspection technique for detection and localization of reflectors in a realistic configuration. Although signal response amplitudes vary with the addition of the knuckle geometry between the transmitting transducer and machined reflectors, the enhancements provided by SAFT processing appears to compensate for these effects and offer a strong basis for developing an optimized inspection protocol for the knuckle region and beyond. The additional capability of utilizing T-SAFT when fully optimized for the sizing application should add the final dimension to an effective and robust inspection solution.

The examination frequency of $3.5 \mathrm{MHz}$ provided good resolution and suitable penetration over the long path lengths. Data acquired from examinations on the mock-up show that the inspection technique is capable of detecting and localizing machined reflectors, over long path lengths and around the knuckle region curvature. Sizing of the reflector depth was not examined in great detail; however, the T-SAFT data indicates the ability to size shallow machined reflectors (with depths greater than $4.6 \mathrm{~mm}$ ) within the established tolerance of $\pm 1.27-\mathrm{mm}$ criteria as it is presently defined.

\section{Deployment Methods for SAFT/T-SAFT Technology}

Deployment of the SAFT/T-SAFT system can utilize conventional robotic crawler technology. Magnetic-wheeled crawlers, which provide a reliable and effective method 
for placing the ultrasonic scanning package in the areas of interest, are commercial off the shelf. Modification to include a scanning bridge for the ultrasonic transducers is necessary, as well as integration of the hardware to the SAFT/T-SAFT computer control system. Figure 5.1 provides a graphic display of a magnetic-wheeled crawler simulating the scanning of a double shell tank knuckle region. The crawler will have an on-board camera system or laser-terrain-following equipment, which can provide accurate directional information for the scan pattern. The weld bead between the knuckle and the lowest shell course will provide a reliable circumferential fiducial for the crawler to follow, allowing scanning from either above or below this weld bead. Control of the crawler and scanning equipment will be conducted remotely from a control station located near the waste tank.

PNNL considered other types of deployment systems, which may provide alternative scanning capabilities. These include:

a) Robotic crawler, travels in the annulus region on the secondary tank bottom. This crawler would require a robot arm to place transducers in the proper orientation on the

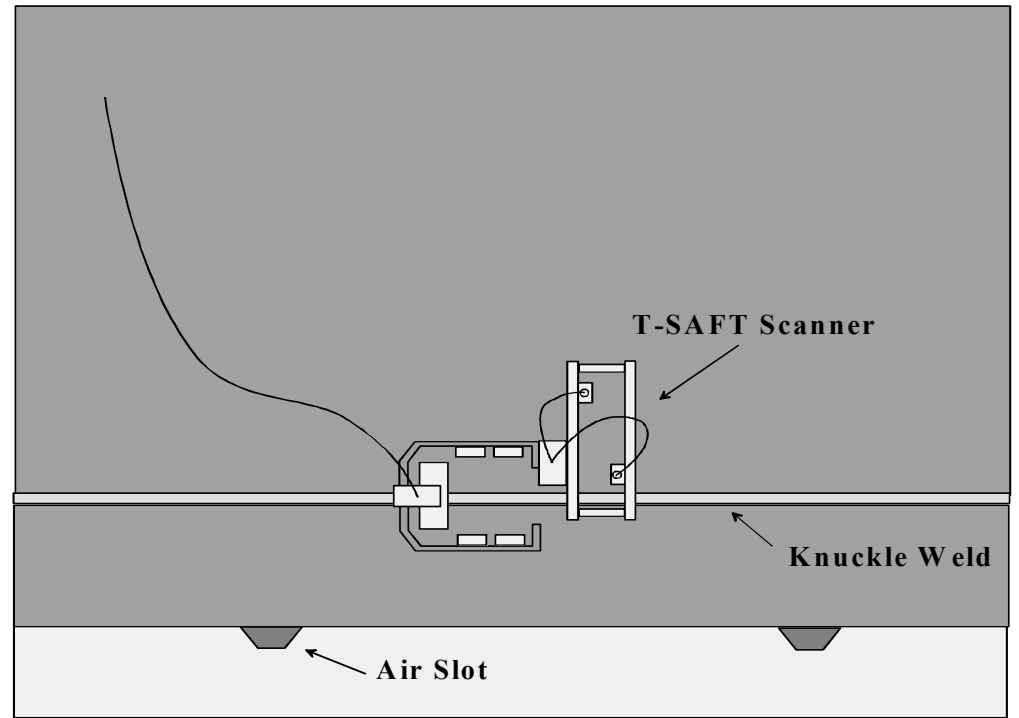

Figure 5.1 SAFT/T-SAFT Deployment Method primary tank knuckle. Since T-SAFT requires the use of two transducers moving simultaneously in opposite directions, the platform and associated mechanics would be difficult to design and may be cost prohibitive.

b) Robotic wall walker which travels the annulus region by utilizing the primary and secondary tank walls. A robot originally designed for use in Hanford's double shell tanks was built and tested here at Hanford during the early 1990's. The robot was not used for examination of the Hanford double shell tanks and the program utilized a magnetic-wheeled crawler instead.

c) Robotic wall walker utilizing suction cups. Wall crawlers that use suction cups to adhere to the tank wall have been designed. Due to the corrosion on the tank walls, which could cause the robot to fall off the wall, and the cost for an operational readiness review of this type of crawler, a system of this nature may be cost prohibitive.

Lessons learned from the current on-going double shell tank inspections point to a magnetic-wheeled crawler as a preferred method for deployment of the SAFT/TSAFT system. Modifications should be straightforward and cost effective. 


\section{Conclusions}

A conclusion based on analyses of the results of the examinations conducted provide good engineering data establishing the proof-of-principle effectiveness for utilizing a combination of pulse-echo SAFT and tandem-SAFT inspection methodologies to the problem of flaw detection, localization and sizing in the knuckle region and beyond. The preliminary studies using pulse-echo, $70^{\circ}$ shear wave inspection modality coupled with SAFT processing was successfully demonstrated as an effective examination method for detection and localization of machined reflectors (sawcuts) over a range of depths (2-mm to $12.7-\mathrm{mm}$ deep), and over long path lengths (approximately $1067 \mathrm{~mm}$ ) and beyond the knuckle region. The packet of signals from the reflectors provide redundancy for flaw detection and may provide improved flaw detection performance for flaws that are not perpendicular to the tank wall but may be tilted. A preliminary evaluation of the tandem, $70^{\circ}$ shear wave inspection modality coupled with tandem-SAFT processing was conducted on the mock-up and appears to provide a robust and effective methodology for sizing those indications detected and localized by the pulse-echo technique.

The inspection system used for digitization, amplification and signal conditioning was not optimal for ultrasonically long-range, attenuative inspection environments. With offthe-shelf electronics and transducers, the inspection techniques evaluated in this study were successfully employed and demonstrated even under such adverse conditions as propagating the sound field through a weldment and around the curved knuckle region. The enhancements provided by SAFT processing provide a solid technical basis to guide the future development of an optimized inspection protocol for the knuckle region and beyond. The additional capability of utilizing an optimized T-SAFT in this application for sizing adds the final dimension to achieving an effective and robust inspection solution.

With regard to laboratory examination techniques, the pulse-echo SAFT and tandemSAFT inspection methodologies implemented here were adequate for demonstrating the feasibility of these technologies. However, for this application the entire inspection protocol must be improved in order to efficiently utilize these techniques with a ruggedized, field-ready tank inspection system. In order to take the proposed inspection protocol to the next level, a number of key elements in the system will require optimization and further refinement. All of the essential components that are required to deploy the inspection methodology are defined, including the SAFT processing code, the data acquisition system electronics, the data analysis and visualization platform, and the front-end transducer-scanner-coupling system. Each essential component is discussed with recommendations for improvement in the next section. 
PNNL-13321

\section{Recommendations}

\subsection{Recommendations for Enhancing SAFT Processing Utility}

Of primary importance to the end-user will be the length of time required to process the raw ultrasonic data. Ideally, the SAFT processing code could be re-designed to provide a real-time or near real-time output of processed data. This output would then be ported to a visualization platform for near-real-time display as the data was being acquired. PNNL staff presently operate SAFT in a post-processing mode where data is processed and imaged after acquisition of raw ultrasonic data. For the data files acquired in this study, the processing times ranged from minutes to nearly four hours or more, depending on the processing parameters applied and scan parameters used. An effort is recommended to support real-time SAFT processing, and will require both software and hardware engineering.

With regard to the processing algorithms used for pulse-echo and tandem SAFT, there are other issues that need to be addressed as well. When processing very long multi-V path lengths in the pulse-echo mode, the SAFT processing algorithm does not allow the user to process the data with a beam processing angle of anything greater than $2^{\circ}$. This is due to how the algorithm uses the calculated dimensions of the divergent beam associated with the very long path length and how it applies the limits to the processing cone. In typical examinations, the synthetic focus is enhanced by utilizing a larger beam processing angle. The SAFT algorithm requires changes in order to compensate for this long path length and allow for a larger beam processing angle to be used for computations.

The tandem-SAFT processing algorithm requires changes to the code that will allow multi-V path length data to be acquired without processing the entire data set associated with the complete path length. Minor changes to the code may also be required in order to compensate for high-angle inspections $\left(70^{\circ}\right.$ and greater) and larger transducers where the side-by-side transducer separation is larger.

\subsection{Recommendations for Enhancing Data Acquisition System Utility}

The data acquisition system includes the PC platform, A/D conversion electronics, pulser-receiver electronics, signal conditioning electronics and filters, and the front-end scanner-transducer-coupling system. Any field-ready and reliable inspection system must be built upon a robust structural framework for data acquisition. Of primary importance to the entire system is the PC platform on which the inspection system resides. It is recommended that the examination system utilize a state-of-the-art, highspeed, dual-processor PC unit for simultaneous operation of the scanner-motor drivers and data acquisition. In order to enhance the laboratory-based system used in this study, a high-speed, 12-bit A/D converter with expanded memory capability should be an integral part of the prototype inspection system. This will significantly improve the 
systems data acquisition speed and dynamic range, and will provide a more efficient method for invoking linear waveform averaging in order to reduce random and electronic white noise introduced by amplifiers and other environmental sources. The pulserreceiver electronics should be optimized to provide a short-duration spike excitation pulse with low-noise amplification over a range of 0-100 dB. Specialized signal conditioning electronics should be utilized for bandpass filtering of the received ultrasonic echoes about the nominal examination frequency of $3.5 \mathrm{MHz}$, in order to condition and filter the signal and reduce extraneous noise sources above and below the center frequency of the transducers. Special high-bandwidth (broadband) transducers should be utilized in order to provide enhanced time-domain resolution between closely spaced echoes. Finally, the scanning system should accommodate low-noise scanning at speeds on the order of 51-102 mm per second. Meeting these recommendations will greatly enhance the effectiveness of the examination method.

\subsection{Recommendations for Enhancing Data Analysis and Visualization}

Utilizing SAFT processing in its present state requires that the operator be well trained in the use of the processing parameters as well as the analysis and visualization platform provided by the code. Unlike commercially available ultrasonic imaging software (i.e., SONIX, P-Scan, etc.) the SAFT processed file output requires the operator to be familiar with A-scan, B-scan (side and end planar views) and C-scan views, as well as projected views. These two-dimensional color representations can be difficult to interpret to the untrained eye. The tools used to properly image and size a reflector are not intuitively obvious and can take weeks to train even qualified NDE inspectors. It is highly recommended that an effort be supported to develop an analysis and visualization platform that utilizes the SAFT output and provides a three-dimensional display that is user friendly and allows for quick and reliable detection and sizing procedures.

\subsection{Recommendations for Prototype Examination System Development, Field Testing and Performance Demonstration}

In order to fabricate, test and demonstrate a field-ready prototype examination system for effective and reliable inspection of the knuckle region and beyond, a two-year effort is recommended. During the first year (FY 01), software enhancements (analysis and visualization tools), changes to the SAFT and T-SAFT algorithms, hardware procurements and system configuration will be conducted. Also during FY 01, sizing criteria will be established and system resolution will be defined by utilizing a set of calibrated reflectors. During year two (FY 02), inspection procedures will be finalized, and field-testing and performance demonstration of the system will be performed. Early in year two, it is proposed that a realistic set of fatigue cracks be fabricated and inserted into the mockup for use in the performance demonstration. 
PNNL-13321

\section{References}

Hall, T. E., L. D. Reid, and S. R. Doctor. 1988. The SAFT-UT Real-Time Inspection System - Operational Principles and Implementation, NUREG/CR-5075. U.S. Nuclear Regulatory Commission, Washington, D.C.

Pfluger, D. C. 1994. Double-Shell Tank Ultrasonic Inspection Plan, WHC-SD- WMAP-019, Westinghouse Hanford Company, Richland, Washington.

Pfluger, D. C. 1995. Double-Shell Tank Ultrasonic Inspection Performance Specification, WHC-S-4108, Westinghouse Hanford Company, Richland, Washington.

Shurrab, M. S., M. D. Thomson, J. R. Friley, M. R. Garnich, M. W. Rinker and F. A. Simonen. 1991. Parametric Studies to Support Inspection Criteria of the Hanford Site Double-Shell Waste Storage Tanks, WHC-EP-0508, Westinghouse Hanford Company, Richland, Washington. 
PNNL-13321

\section{Distribution}

No. of

$\underline{\text { Copies }}$

Offsite

4 DOE/Office of Scientific and

Technical Information \&

Information Release

1 DOE Office of Science and

Technology

Kurt Gerdes

1154 Cloverleaf Building

19901 Germantown Road

Germantown, MD 20874-1290

Onsite

8 Tanks Focus Area Technical Team

B. J. Williams K9-69

1 Tanks Focus Area Program Manager

T. P. Pietrok K8-50

1 Tanks Focus Area - Safety

M. T. Terry K9-91

$1 \quad$ Hanford Site
E. J. Cruz
H6-60

13 Pacific Northwest National Laboratory

J. B. Colson (1)

K5-25

A. A. Diaz (3) K5-26

S. R. Doctor (1) K5-26

A. F. Pardini (5) K5-26

G. J. Posakony (1) K5-26

G. J. Schuster (1) K5-26

T. T. Taylor (1) K5-26

Distr. 1 